\title{
Projective integral models of Shimura varieties of Hodge type with compact factors
}

\author{
Adrian Vasiu, U of Arizona, 09/01/04, revision 01/30/06
}

\begin{abstract}
Let $(G, X)$ be a Shimura pair of Hodge type such that $G$ is the MumfordTate group of some elements of $X$. We assume that for each simple factor $G_{0}$ of $G^{\text {ad }}$ there exists a simple factor of $G_{0 \mathbb{R}}$ which is compact. Let $N \geq 3$. We show that for many compact, open subgroups $K$ of $G\left(\mathbb{A}_{f}\right)$, the Shimura variety $\operatorname{Sh}(G, X) / K$ has a projective integral model $\mathcal{N}$ over $\mathbb{Z}\left[\frac{1}{N}\right]$ which is a finite scheme over a certain Mumford moduli scheme $\mathcal{A}_{g, 1, N}$. This result represents significant progress towards the proof of a conjecture of Morita. If $\mathcal{N}$ is smooth over $\mathbb{Z}\left[\frac{1}{N}\right]$, then it is a Néron model of its generic fibre. In this way one gets in arbitrary mixed characteristic, the very first examples of general nature of projective Néron models whose generic fibres are not finite schemes over abelian varieties.
\end{abstract}

Key words: abelian and semiabelian schemes, Mumford-Tate groups, Shimura varieties, integral models, and Néron models.

MSC 2000: Primary 11G10, 11G18, 14F30, 14G35, 14K10, 14K15, and 14J20.

\section{$\S 1$. Introduction}

Let $\mathbb{S}:=\operatorname{Res}_{\mathbb{C} / \mathbb{R}} \mathbb{G}_{m \mathbb{C}}$ be the two dimensional torus over $\mathbb{R}$ with the property that $\mathbb{S}(\mathbb{R})$ is the multiplicative group of non-zero complex numbers. Let $E$ be a number field. Let $O_{E}$ be the ring of integers of $E$. We fix an embedding $i_{E}: E \hookrightarrow \mathbb{C}$. Let $A$ be an abelian variety over $E$. Let $W_{A}:=H_{1}(A(\mathbb{C}), \mathbb{Q})$ be the first Betti homology group of the complex manifold $A(\mathbb{C})$ with coefficients in $\mathbb{Q}$. Let $h_{A}: \mathbb{S} \rightarrow \mathrm{GL}_{W_{A} \otimes_{\mathbb{Q}} \mathbb{R}}$ be the homomorphism that defines the Hodge $\mathbb{Q}$-structure on $W_{A}$. Let $H_{A}$ be the Mumford-Tate group of $A_{\mathbb{C}}$. We recall that $H_{A}$ is a reductive group over $\mathbb{Q}$ and that $H_{A}$ is the smallest subgroup of $\mathrm{GL}_{W_{A}}$ with the property that $h_{A}$ factors through $H_{A \mathbb{R}}$, cf. [8], Propositions 3.6 and 3.4. Let $H_{A}^{\text {ad }}$ be the adjoint group of $H_{A}$.

1.1. Definition. We say the abelian variety $A$ has compact factors, if for each simple factor $H_{0}$ of $H_{A}^{\text {ad }}$ there exists a simple factor of $H_{0 \mathbb{R}}$ which is compact.

It is conjectured (see [21], page 437 and [27], Conjecture 3.1.3) that if the $\mathbb{Q}$-rank of $H_{A}^{\mathrm{ad}}$ is 0 , then there exists a finite field extension $E_{1}$ of $E$ such that $A_{E_{1}}$ extends to an abelian scheme over $O_{E_{1}}$ (i.e., such that $A_{E_{1}}$ has good reduction with respect to all finite primes of $E_{1}$ ). See [27] for other equivalent forms of this conjecture. Following [27], below we will refer to this conjecture as the Morita conjecture. We note down that if $A$ has compact factors, then each simple factor of $H_{A}^{\text {ad }}$ has $\mathbb{Q}$-rank 0 and thus $H_{A}^{\text {ad }}$ itself has $\mathbb{Q}$-rank 0. The goals of this paper are: (i) to prove the Morita conjecture under the assumption that $A$ has compact factors, and (ii) to reformulate and apply this result to integral models of Shimura varieties of Hodge type.

1.2. Basic Theorem. Suppose A has compact factors. Then there exists a finite field extension $E_{1}$ of $E$ such that $A_{E_{1}}$ extends to an abelian scheme over $O_{E_{1}}$. 
Morita conjecture was first checked in some cases involving abelian varieties of PEL type (see [21]; see also [15], end of $\S 5$ ). We recall that $A$ is of PEL type, if $A_{\mathbb{C}}$ has a polarization $\lambda$ such that the derived group of $H_{A}$ is also the derived group of the intersection of $\operatorname{GSp}\left(W_{A}, \psi\right)$ with the double centralizer of $H_{A}$ in $\mathrm{GL}_{W_{A}}$ (here $\psi$ is the alternating form on $W_{A}$ defined by $\lambda$ and PEL stands for polarization, endomorphisms, and level structures). Some of the cases presented in [21] are not covered by the Basic Theorem.

New cases of the validity of the Morita conjecture are provided in [25] and [27]. The results [27], Propositions 4.2.2, 4.2.4, and 4.2.10 cover [25]; these results are also either covered by [27], Proposition 4.2.13 (equivalently, by Example 4.2.2 of this paper) or involve special cases when there exists a good prime $p \in \mathbb{N}$ for which a certain combinatorial condition on the natural action of $\operatorname{Gal}\left(\mathbb{Q}_{p}\right)$ on the set of simple factors of $H_{A \overline{\mathbb{Q}_{p}}}^{\text {ad }}$ holds (see [27], Example 4.2.11 for such a concrete special case). Such good primes $p$ exist only if $A$ has compact factors and each simple factor $H_{0}$ of $H_{A}^{\text {ad }}$ is "simple enough" (like when $H_{0 \mathbb{R}}$ has only one simple, non-compact factor). Good primes $p$ do not exist if there exists one simple factor $H_{0}$ of $H_{A}^{\text {ad }}$ such that the group $H_{0 \mathbb{R}}$ : either (a) has more simple, non-compact factors than simple, compact factors or (b) it is a Weil restriction $\operatorname{Res}_{F_{0} / \mathbb{Q}} \tilde{H}_{0}$, where $\tilde{H}_{0}$ is an absolutely simple, adjoint group over some "arithmetically complicated" totally real number field $F_{0}$. Thus the results [27], Propositions 4.2.2, 4.2.4, 4.2.10, and 4.2.13 are particular cases of the combination of the Basic Theorem and Example 4.2.2.

Basic Theorem, Example 4.2.2, and (the situations that can be reduced to) [15], end of $\S 5$, form all (general) cases in which the Morita conjecture is presently known to hold. Example 4.2.2 and [15], end of $\S 5$ pertain only to abelian varieties $A$ over $E$ for which, up to a replacement of $E$ by a finite field extension of it, there exists an abelian variety $B$ over $E$ that is of PEL type and that has the property that the three adjoint groups $H_{A}^{\text {ad }}, H_{B}^{\text {ad }}$, and $H_{A \times_{E} B}^{\text {ad }}$ are isomorphic. In particular, Example 4.2.2 reproves the Morita conjecture in the case when there exists a prime $p \in \mathbb{N}$ such that the $\mathbb{Q}_{p}$-rank of $H_{A \mathbb{Q}_{p}}^{\text {ad }}$ is 0 .

The methods we use to prove the Basic Theorem pertain to Shimura varieties and rely on the constructions of [7], Proposition 2.3.10 and [30], Subsections 6.5 and 6.6. To outline the methods, in this paragraph we assume $A$ has compact factors. We use the mentioned constructions in order to show that for a given prime $p \in \mathbb{N}$, up to a replacement of $E$ by a finite field extension of it, there exists an abelian variety $B$ over $E$ which has the following two properties: (i) the three adjoint groups $H_{A}^{\text {ad }}, H_{B}^{\text {ad }}$, and $H_{A \times_{E} B}^{\text {ad }}$ are isomorphic, and (ii) the monomorphism $H_{B} \hookrightarrow \mathrm{GL}_{W_{B}}$ is "manageable enough" so that we can check based on [10] and [8] that $B$ has good reduction with respect to all prime of $E$ that divide $p$ (see $\S 3$ and 4.1). Due to (i) and the good reduction part of (ii), there exists a finite field extension $E_{p}$ of $E$ such that $A_{E_{p}}$ has good reduction with respect to all primes of $E_{p}$ that divide $p$ (see [27], Proposition 4.1.2 and 4.1). As the field $E_{1}$ mentioned in the Basic Theorem, we can take the composite field of a suitable finite set of such fields $E_{p}$ (see 4.1).

Such "extra" abelian varieties $B$, were first considered in [32] in connection to the Mumford-Tate conjecture for $A$ and in [27] in connection to the Morita conjecture for $A$.

In $\S 2$ we review basic properties of Shimura varieties. In $\S 3$ we include a construction that is the very essence of the proof of the Basic Theorem and which in fact proves the Basic Theorem under some additional hypotheses. In 4.1 we prove the Basic Theorem. In 4.2 we include two examples meant to make the connection between our results and the 
results of [27]. In 4.3 we reformulate the Basic Theorem in terms of integral models of Shimura varieties of Hodge type. In 4.4 we apply 4.3 to provide new examples of general nature of Néron models in the sense of [3], page 12. In 4.5 and 4.6 we apply 4.3 to correct an error in [30], Remark 6.4.1.1 2) and thus implicitly in [30], Subsubsection 6.4.11, for the cases to which the Basic Theorem applies (i.e., for Shimura pairs of preabelian type that have compact factors in a sense analogous to 1.1 ; see 2.2 and 2.4 for precise definitions).

Acknowledgment. We would like to thank U of Arizona for providing us with good conditions in which to write this work.

\section{$\S 2$. Preliminaries}

In 2.1 we gather different notations to be often used in the rest of the paper. See 2.2 for generalities on Shimura pairs and varieties. See 2.3 for simple properties that pertain to polarizations of $A$ and to the reductive group $H_{A}$. See 2.4 for the Shimura pairs naturally associated to $A$. See 2.5 for smooth toroidal compactifications. See 2.6 and 2.7 for two results that pertain to an equivalent form of 1.2 to be stated explicitly in 4.3 .

2.1. Notations and conventions. A reductive group $H$ over a field $k$ is assumed to be connected. Let $Z(H), H^{\text {der }}$, and $H^{\text {ad }}$ denote the center, the derived group, and the adjoint group (respectively) of $H$. We have $H^{\text {ad }}=H / Z(H)$. Let $Z^{0}(H)$ be the maximal subtorus of $Z(H)$. Let $H^{\text {sc }}$ be the simply connected semisimple group cover of $H^{\text {der }}$. See [3], Subsection 7.6 for the Weil restriction of scalars functor $\operatorname{Res}_{k_{1} / k}$, where $k_{1}$ is a separable, finite field extension of $k$. For a free module $M$ of finite rank over a commutative ring with unit $R$, let $M^{*}:=\operatorname{Hom}_{R}(M, R)$ and let $\mathrm{GL}_{M}$ be the group scheme over $R$ of linear automorphisms of $M$. If $\psi$ is a perfect alternating form on $M$, then $\operatorname{GSp}(M, \psi)$ is viewed as a reductive group scheme over $R$. If $*$ or $*_{R}$ (resp. $*_{+}$with + as an arbitrary index different from $R$ ) is either an object or a morphism of the category of $\operatorname{Spec}(R)$-schemes, let $*_{U}\left(\right.$ resp. $\left.*_{+}\right)$be its pull back via an affine morphism $\operatorname{Spec}(U) \rightarrow \operatorname{Spec}(R)$. Let $\overline{\mathbb{Q}}$ be the algebraic closure of $\mathbb{Q}$ in $\mathbb{C}$. We always use the notations of the first paragraph of $\S 1$.

2.2. On Shimura pairs. A Shimura pair $(G, X)$ consists of a reductive group $G$ over $\mathbb{Q}$ and a $G(\mathbb{R})$-conjugacy class $X$ of homomorphisms $\mathbb{S} \rightarrow G_{\mathbb{R}}$ that satisfy Deligne's axioms of [7], Subsubsection 2.1.1: the Hodge $\mathbb{Q}$-structure on $\operatorname{Lie}(G)$ defined by any $x \in X$ is of type $\{(-1,1),(0,0),(1,-1)\}$, Ad $\circ x(i)$ defines a Cartan involution of $\operatorname{Lie}\left(G_{\mathbb{R}}^{\text {ad }}\right)$, and no simple factor of $G^{\text {ad }}$ becomes compact over $\mathbb{R}$. Here Ad : $G_{\mathbb{R}} \rightarrow \mathrm{GL}_{\mathrm{Lie}\left(G_{\mathbb{R}}^{\text {ad }}\right)}$ is the adjoint representation. These axioms imply that $X$ has a natural structure of a hermitian symmetric domain (see [7], Corollary 1.1.17). Similarly to 1.1 , we say $(G, X)$ has compact factors, if for each simple factor $G_{0}$ of $G^{\text {ad }}$ there exists a simple factor of $G_{0 \mathbb{R}}$ which is compact (thus $G_{0 \mathbb{R}}$ has at least one simple, non-compact factor and at least one simple, compact factor). For generalities on Shimura pairs and varieties and on their types, we refer to [6], [7], [18], [19], and [30], Subsections 2.2 to 2.5. To $(G, X)$ it is associated naturally a number field $E(G, X)$, called the reflex field of $(G, X)$ (see [6], [7], and [17]).

Let $\mathbb{A}_{f}:=\widehat{\mathbb{Z}} \otimes_{\mathbb{Z}} \mathbb{Q}$ be the ring of finite adèles of $\mathbb{Q}$. For $K$ a compact, open subgroup of $G\left(\mathbb{A}_{f}\right)$, let $\operatorname{Sh}(G, X)_{\mathbb{C}} / K:=G(\mathbb{Q}) \backslash X \times G\left(\mathbb{A}_{f}\right) / K$; it is a finite disjoint union of quotients 
of a connected component of $X$ by arithmetic subgroups of $G(\mathbb{Q})$. A theorem of Baily and Borel says that $\operatorname{Sh}(G, X)_{\mathbb{C}} / K$ has a canonical structure of a normal, quasi-projective $\mathbb{C}$-scheme (see [1], Theorem 10.11) which is smooth if $K$ is small enough. Thus the projective limit $\operatorname{Sh}(G, X)_{\mathbb{C}}$ of all the normal, quasi-projective $\mathbb{C}$-schemes $\operatorname{Sh}(G, X)_{\mathbb{C}} / K$ 's, has a canonical structure of a regular $\mathbb{C}$-scheme. The Shimura variety $\operatorname{Sh}(G, X)$ is identified with the canonical model over $E(G, X)$ of the $\mathbb{C}$-scheme $\operatorname{Sh}(G, X)_{\mathbb{C}}$ (see [6], [7], [17], [19], and $[20])$. We have a natural right action of $G\left(\mathbb{A}_{f}\right)$ on the $E(G, X)$-scheme $\operatorname{Sh}(G, X)$ which is continuous in the sense of [7], Subsubsection 2.7.1. In particular, the quotient $\operatorname{Sh}(G, X) / K$ of $\operatorname{Sh}(G, X)$ through $K$ is a normal, quasi-projective $E(G, X)$-scheme which is "the best arithmetic" model of $\operatorname{Sh}(G, X)_{\mathbb{C}} / K$.

2.3. Simple properties. The notion of good reduction of an abelian variety over the field of fractions of a discrete valuation ring, is stable under isogenies. Up to a replacement of $E$ by a finite field extension of it, $A$ is isogenous to a principally polarized abelian variety over $E$ (cf. [23], $\S 23$, Corollary 1). Thus, based on the last two sentences, to prove 1.2 (and for the rest of the paper) we can assume that $A$ has a principal polarization $\lambda_{A}$.

2.3.1. Fact. If $H_{A}$ is a torus (i.e., if the adjoint group $H_{A}^{\mathrm{ad}}$ is trivial), then there exists a finite field extension $E_{1}$ of $E$ such that $A_{E_{1}}$ extends to an abelian scheme over $O_{E_{1}}$.

Proof: Let $p \in \mathbb{N}$ be a prime. All elements of $H_{A}\left(\mathbb{Q}_{p}\right)$ are semisimple and therefore the group $H_{A}\left(\mathbb{Q}_{p}\right)$ has no non-trivial unipotent element. Thus this well known Fact is a very particular case of [27], Theorem 1.6.1.

2.4. Shimura pairs associated to $A$. Let $L_{A}:=H_{1}(A(\mathbb{C}), \mathbb{Z})$; it is a $\mathbb{Z}$-lattice of $W_{A}$. Let $\psi_{A}: L_{A} \otimes_{\mathbb{Z}} L_{A} \rightarrow \mathbb{Z}$ be the perfect, alternating form on $L_{A}$ induced by $\lambda_{A}$. If $W_{A} \otimes_{\mathbb{Q}} \mathbb{C}=$ $F_{A}^{-1,0} \oplus F_{A}^{0,-1}$ is the Hodge decomposition defined by $h_{A}$, let $\mu_{A}: \mathbb{G}_{m \mathbb{C}} \rightarrow \mathrm{GL}_{W_{A} \otimes_{\mathbb{Q}}}$ be the Hodge cocharacter that fixes $F_{A}^{0,-1}$ and that acts via the identical character of $\mathbb{G}_{m} \mathbb{C}$ on $F_{A}^{-1,0}$. We denote also by $h_{A}: \mathbb{S} \rightarrow H_{A \mathbb{R}}$ and $\mu_{A}: \mathbb{G}_{m \mathbb{C}} \rightarrow H_{A \mathbb{C}}$ the natural factorizations of $h_{A}$ and $\mu_{A}$ (respectively). Let $X_{A}$ be the $H_{A}(\mathbb{R})$-conjugacy class of $h_{A}: \mathbb{S} \rightarrow H_{A \mathbb{R}}$. Let $S_{A}$ be the $\operatorname{GSp}\left(W_{A}, \psi_{A}\right)(\mathbb{R})$-conjugacy class of the homomorphism $\mathbb{S} \rightarrow \operatorname{GSp}\left(W_{A}, \psi_{A}\right)_{\mathbb{R}}$ defined by $h_{A}$. It is well known that the pairs $\left(H_{A}, X_{A}\right)$ and $\left(\operatorname{GSp}\left(W_{A}, \psi_{A}\right), S_{A}\right)$ are Shimura pairs and that we have an injective map $f_{A}:\left(H_{A}, X_{A}\right) \hookrightarrow\left(\operatorname{GSp}\left(W_{A}, \psi_{A}\right), S_{A}\right)$ of Shimura pairs (see [6], [7], [17], and [19]). The Shimura variety $\operatorname{Sh}\left(\operatorname{GSp}\left(W_{A}, \psi_{A}\right), S_{A}\right)$ is called a Siegel modular variety. A Shimura pair that admits an injective map into a Shimura pair that defines a Siegel modular variety, is called a Shimura pair of Hodge type; thus $\left(H_{A}, X_{A}\right)$ is a Shimura pair of Hodge type.

Let $E_{A}:=E\left(H_{A}, X_{A}\right)$. Let $g:=\operatorname{dim}(A)$. Let $h_{A}^{\text {ad }}: \mathbb{S} \rightarrow H_{A \mathbb{R}}^{\text {ad }}$ be the composite of $h_{A}$ with the natural epimorphism $H_{A \mathbb{R}} \rightarrow H_{A \mathbb{R}}^{\text {ad }}$. Let $X_{A}^{\text {ad }}$ be the $H_{A}^{\text {ad }}(\mathbb{R})$-conjugacy class of $h_{A}^{\text {ad }}$. The pair $\left(H_{A}^{\mathrm{ad}}, X_{A}^{\mathrm{ad}}\right)$ is called the adjoint Shimura pair of $\left(H_{A}, X_{A}\right)$.

Similarly we define the adjoint Shimura pair $\left(G^{\text {ad }}, X^{\text {ad }}\right)$ of an arbitrary Shimura pair $(G, X)$. We recall that $(G, X)$ is said to be of preabelian type, if $\left(G^{\text {ad }}, X^{\text {ad }}\right)$ is isomorphic to $\left(H_{A}^{\mathrm{ad}}, X_{A}^{\mathrm{ad}}\right)$ for some abelian variety $A$ over a number field $E$ (see [18], [30], etc.).

Let $N \in \mathbb{N} \backslash\{1,2\}$. Let $\psi_{A, N}: L_{A} / N L_{A} \otimes_{\mathbb{Z} / N \mathbb{Z}} L_{A} / N L_{A} \rightarrow \mathbb{Z} / N \mathbb{Z}$ be the reduction $\bmod N$ of $\psi_{A}$. Let $\left(C, \lambda_{C}\right)$ be a principally polarized abelian scheme of relative dimension 
$g$ over a $\mathbb{Z}\left[\frac{1}{N}\right]$-scheme $Y$. Let $\lambda_{C[N]}: C[N] \times_{Y} C[N] \rightarrow \mu_{N Y}$ be the Weil pairing induced by $\lambda_{C}$. By a level- $N$ symplectic similitude structure of $\left(C, \lambda_{C}\right)$ we mean an isomorphism $\kappa:\left(L_{A} / N L_{A}\right)_{Y} \stackrel{\sim}{\rightarrow} C[N]$ between finite, étale group schemes over $Y$, such that there exists an element $\nu \in \mu_{N Y}(Y)$ with the property that for all points $a, b \in\left(L_{A} / N L_{A}\right)_{Y}(Y)$ we have an identity $\nu^{\psi_{A, N}(a \otimes b)}=\lambda_{C[N]}(\kappa(a), \kappa(b))$ between elements of $\mu_{N Y}(Y)$.

Let $\mathcal{A}_{g, 1, N}$ be the moduli scheme over $\mathbb{Z}\left[\frac{1}{N}\right]$ that parameterizes principally polarized abelian schemes which are of relative dimension $g$ and which are equipped with a level- $N$ symplectic similitude structure, cf. [22], Theorems 7.9 and 7.10 adapted naturally to the case of level- $N$ symplectic similitude structures (instead of only level- $N$ structures). Let $\left(\mathcal{A}, \lambda_{\mathcal{A}}\right)$ be the universal principally polarized abelian scheme over $\mathcal{A}_{g, 1, N}$.

Let $K(N):=\left\{h \in \operatorname{GSp}\left(L_{A}, \psi_{A}\right)(\widehat{\mathbb{Z}}) \mid h \bmod N\right.$ is the identity $\}$. Let $K_{A}(N):=$ $K(N) \cap H_{A}\left(\mathbb{A}_{f}\right)$. It is known that we can identify $\operatorname{Sh}\left(\operatorname{GSp}\left(W_{A}, \psi_{A}\right), S_{A}\right) / K(N)=\mathcal{A}_{g, 1, N_{\mathbb{Q}}}$, see [6], Proposition 4.17. To $f_{A}$ corresponds a finite (functorial) morphism of $\mathbb{Q}$-schemes

$$
f_{A}(N): \operatorname{Sh}\left(H_{A}, X_{A}\right) / K_{A}(N) \rightarrow \operatorname{Sh}\left(\operatorname{GSp}\left(W_{A}, \psi_{A}\right), S_{A}\right)_{E_{A}} / K(N)
$$

which over $\mathbb{C}$ is obtained from the embedding $X_{A} \times H_{A}\left(\mathbb{A}_{f}\right) \hookrightarrow S_{A} \times \operatorname{GSp}\left(W_{A}, \psi_{A}\right)\left(\mathbb{A}_{f}\right)$ between complex spaces via a natural passage to quotients, cf. [6], Corollary 5.4. Let $\mathcal{N}_{N}:=\mathcal{N}_{A, N}$ be the normalization of $\mathcal{A}_{g, 1, N}$ in the ring of fractions of $\operatorname{Sh}\left(H_{A}, X_{A}\right) / K_{A}(N)$; it is an $O_{E_{A}}\left[\frac{1}{N}\right]$-scheme. Let $\left(\mathcal{B}, \lambda_{\mathcal{B}}\right)$ be the pull back of $\left(\mathcal{A}, \lambda_{\mathcal{A}}\right)$ to $\mathcal{N}_{N}$.

2.4.1. On factors. Let $\mu_{A}^{\text {ad }}: \mathbb{G}_{m \mathbb{C}} \rightarrow H_{A \mathbb{C}}^{\text {ad }}$ be the cocharacter defined naturally by $\mu_{A}$. For an arbitrary simple factor $H_{t}$ of $H_{A}^{\text {ad }}$, let $\mu_{t}: \mathbb{G}_{m \overline{\mathbb{Q}}} \rightarrow H_{t \overline{\mathbb{Q}}}$ be a cocharacter whose extension to $\mathbb{C}$ is $H_{t}(\mathbb{C})$-conjugate to the cocharacter of $H_{t \mathbb{C}}$ defined naturally by $\mu_{A}^{\text {ad }}$. Let $h_{A t}: \mathbb{S} \rightarrow H_{t \mathbb{R}}$ be the homomorphism defined naturally by $h_{A}$. Until $\S 4$, we denote by $H_{0}$ a fixed simple factor of $H_{A}^{\text {ad }}$. Thus we will speak about $\mu_{0}: \mathbb{G}_{m \overline{\mathbb{Q}}} \rightarrow H_{0 \overline{\mathbb{Q}}}$ and $h_{A 0}: \mathbb{S} \rightarrow H_{0 \mathbb{R}}$.

2.4.2. On complex points. We have $\operatorname{Sh}\left(H_{A}, X_{A}\right)(\mathbb{C})=H_{A}(\mathbb{Q}) \backslash X_{A} \times H_{A}\left(\mathbb{A}_{f}\right)$, cf. [7], Proposition 2.1.10 and Corollary 2.1.11. Let $u:=[x, h] \in \operatorname{Sh}\left(H_{A}, X_{A}\right)(\mathbb{C})$, where $x \in X_{A}$ and $h \in H_{A}\left(\mathbb{A}_{f}\right)$. Let $W_{A} \otimes_{\mathbb{Q}} \mathbb{C}=F_{x}^{-1,0} \oplus F_{x}^{0,-1}$ be the Hodge decomposition defined by $x: \mathbb{S} \rightarrow H_{A \mathbb{R}}$ and let $L_{h}$ be the $\mathbb{Z}$-lattice of $W_{A}$ with the property that $h\left(L_{A} \otimes_{\mathbb{Z}} \widehat{\mathbb{Z}}\right)=L_{h} \otimes_{\mathbb{Z}} \widehat{\mathbb{Z}}$. We denote also by $u \in \operatorname{Sh}\left(H_{A}, X_{A}\right) / K_{A}(N)(\mathbb{C})$ the composite of $u$ with the natural quotient map $\operatorname{Sh}\left(H_{A}, X_{A}\right) \rightarrow \operatorname{Sh}\left(H_{A}, X_{A}\right) / K_{A}(N)$. The complex torus associated to the abelian variety $B_{u}:=u^{*}(\mathcal{B})$ is $L_{h} \backslash W_{A} \otimes_{\mathbb{Q}} \mathbb{C} / F_{x}^{0,-1}$. The principal polarization $u^{*}\left(\lambda_{\mathcal{B}}\right)$ of $B_{u}$ is uniquely determined by the property that it induces a perfect alternating form on $L_{h}$ which is a rational multiple of $\psi_{A}$ and which is a polarization of the Hodge $\mathbb{Q}$-structure on $W_{A}$ defined by $x$, cf. [30], Subsection 4.1.

Let $C_{A}$ be the centralizer of $H_{A}$ in $\operatorname{End}\left(W_{A}\right)$. Due to Riemann's theorem (see [6], Theorem 4.7) we can naturally view $C_{A}$ : (i) as a $\mathbb{Q}$-algebra of $\mathbb{Q}$-endomorphisms of any such pull pull back $B_{u}$ of $\mathcal{B}$, and (ii) as $\operatorname{End}\left(A_{\mathbb{C}}\right) \otimes_{\mathbb{Z}} \mathbb{Q}$. We identify naturally $W_{A}=$ $H_{1}\left(B_{u}(\mathbb{C}), \mathbb{Q}\right)$; such an identification is unique up to isomorphisms $W_{A} \stackrel{\sim}{\rightarrow} W_{A}$ defined by elements of $H_{A}(\mathbb{Q})$. If $p \in \mathbb{N}$ is a prime, then we also identify naturally $W_{A}^{*} \otimes_{\mathbb{Q}} \mathbb{Q}_{p}=$ $H_{e ́ t}^{1}\left(B_{u}, \mathbb{Q}_{p}\right)$. The identification $W_{A}=H_{1}\left(B_{u}(\mathbb{C}), \mathbb{Q}\right)\left(\operatorname{resp} . W_{A}^{*} \otimes_{\mathbb{Q}} \mathbb{Q}_{p}=H_{e ́ t}^{1}\left(B_{u}, \mathbb{Q}_{p}\right)\right)$ is compatible with the natural actions of $C_{A}$ (resp. of the opposite $\mathbb{Q}$-algebra $C_{A}^{\text {opp }}$ of $C_{A}$ ).

2.4.3. Special points. We now assume $x$ is a special point (i.e., the homomorphism $x: \mathbb{S} \rightarrow H_{A \mathbb{R}}$ factors through the extension to $\mathbb{R}$ of a maximal torus $T_{x}$ of $H_{A}$ ). The 
Mumford-Tate group of $B_{u}$ is a reductive subgroup of $T_{x}$ and thus it is a torus. We have $u \in \operatorname{Im}\left(\operatorname{Sh}\left(H_{A}, X_{A}\right)(\overline{\mathbb{Q}}) \rightarrow \operatorname{Sh}\left(H_{A}, X_{A}\right)(\mathbb{C})\right)$, cf. [18], Theorem 1.8 applied to the special pair $\left(T_{x},\{x\}\right)$ of $\left(H_{A}, X_{A}\right)$. The set of all points $u=[x, h] \in \operatorname{Sh}\left(H_{A}, X_{A}\right)(\mathbb{C})$ with $h \in H_{A}\left(\mathbb{A}_{f}\right)$, is Zariski dense in $\operatorname{Sh}\left(H_{A}, X_{A}\right)(\mathbb{C})$ (cf. [6], Proposition 5.2).

2.5. Toroidal compactifications. We consider a smooth, projective, toroidal compactification $\overline{\mathcal{A}}_{g, 1, N}$ of $\mathcal{A}_{g, 1, N}$ over $\mathbb{Z}\left[\frac{1}{N}\right]$ such that the abelian scheme $\mathcal{A}$ over $\mathcal{A}_{g, 1, N}$ extends to a semiabelian scheme $\overline{\mathcal{A}}$ over $\overline{\mathcal{A}}_{g, 1, N}$ (cf. [10], Chapter IV, Theorem 6.7). We have:

(i) The fibres of $\overline{\mathcal{A}}$ over points of the complement of $\mathcal{A}_{g, 1, N}$ in $\overline{\mathcal{A}}_{g, 1, N}$, are semiabelian varieties that are not abelian varieties.

We consider the normalization $\overline{\mathcal{N}}_{N}:=\overline{\mathcal{N}}_{A, N}$ of $\overline{\mathcal{A}}_{g, 1, N}$ in the ring of fractions of $\operatorname{Sh}\left(H_{A}, X_{A}\right) / K_{A}(N)$; it is an $O_{E_{A}}\left[\frac{1}{N}\right]$-scheme. As the morphism $f_{A}(N)$ is finite and as $\mathcal{A}_{g, 1, N}$ is an open, Zariski dense subscheme of $\overline{\mathcal{A}}_{g, 1, N}$, we have:

(ii) The $O_{E_{A}}\left[\frac{1}{N}\right]$-scheme $\mathcal{N}_{N}$ is an open, Zariski dense subscheme of $\overline{\mathcal{N}}_{N}$ and we have $\mathcal{N}_{N E_{A}}=\operatorname{Sh}\left(H_{A}, X_{A}\right) / K_{A}(N)$.

Let $\overline{\mathcal{B}}$ be the pull back of $\overline{\mathcal{A}}$ to $\overline{\mathcal{N}}_{N}$. Thus $\overline{\mathcal{B}}$ is a semiabelian scheme over $\overline{\mathcal{N}}_{N}$ whose restriction to $\mathcal{N}_{N}$ is the abelian scheme $\mathcal{B}$.

2.6. Fact. The following two statements are equivalent:

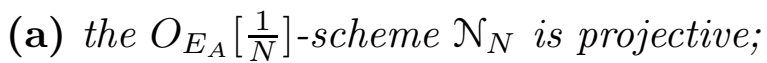

(b) we have $\mathcal{N}_{N}=\overline{\mathcal{N}}_{N}$.

Moreover, if these two statements hold, then there exists a finite field extension $E_{1}$ of $E$ such that $A_{E_{1}}$ extends to an abelian scheme over $O_{E_{1}}\left[\frac{1}{N}\right]$.

Proof: As $O_{E_{A}}$ is an excellent ring (see [16], §34), the scheme $\overline{\mathcal{N}}_{N}$ is a finite $\overline{\mathcal{A}}_{g, 1, N}$-scheme and thus a projective $\mathbb{Z}\left[\frac{1}{N}\right]$-scheme. As $\overline{\mathcal{N}}_{N}$ is an $O_{E_{A}}\left[\frac{1}{N}\right]$-scheme, we get that $\overline{\mathcal{N}}_{N}$ is a projective $O_{E_{A}}\left[\frac{1}{N}\right]$-scheme. But $\mathcal{N}_{N}$ is an open, Zariski dense subscheme of $\overline{\mathcal{N}}_{N}$ and thus (a) is equivalent to (b). To end the proof, it suffices to show that (a) implies the existence of a finite field extension $E_{1}$ of $E$ such that $A_{E_{1}}$ extends to an abelian scheme over $O_{E_{1}}\left[\frac{1}{N}\right]$.

Let $E_{1}$ be a finite field extension of $E$ in $\mathbb{C}$ such that there exists a level- $N$ symplectic similitude structure $\kappa$ of $\left(A, \lambda_{A}\right)_{E_{1}}$ whose pull back to $\mathbb{C}$ is defined by the canonical isomorphism $\left(L_{A} / N L_{A}\right)_{\mathbb{C}} \stackrel{\sim}{\rightarrow} A_{\mathbb{C}}[N]=\left(\frac{1}{N} L_{A} / L_{A}\right)_{\mathbb{C}}$. Let $v_{A}: \operatorname{Spec}\left(E_{1}\right) \rightarrow \mathcal{A}_{g, 1, N}$ be the morphism such that $\left(A, \lambda_{A}\right)_{E_{1}}=v_{A}^{*}\left(\mathcal{A}, \lambda_{\mathcal{A}}\right)$ and the resulting level- $N$ symplectic similitude structure of $\left(A, \lambda_{A}\right)_{E_{1}}$ is $\kappa$. The composite of the morphism $\operatorname{Spec}(\mathbb{C}) \rightarrow \operatorname{Spec}\left(E_{1}\right)$ with $v_{A}$, is the complex point $\left[h_{A}, 1_{W_{A}}\right] \in \operatorname{Im}\left(f_{A}(N)(\mathbb{C})\right)$ (here $1_{W_{A}}$ is the identity element of $\operatorname{GSp}\left(W_{A}, \psi_{A}\right)\left(\mathbb{A}_{f}\right)$. This implies that up to a replacement of $E_{1}$ by a finite field extension of it, $v_{A}$ factors through a morphism $u_{A}: \operatorname{Spec}\left(E_{1}\right) \rightarrow \mathcal{N}_{N}$. As (a) holds, from the valuative criterion of properness we get that $u_{A}$ extends to a morphism $u_{A, N}: \operatorname{Spec}\left(O_{E_{1}}\left[\frac{1}{N}\right]\right) \rightarrow \mathcal{N}_{N}$. Thus the abelian scheme $u_{A, N}^{*}(\mathcal{B})$ over $O_{E_{1}}\left[\frac{1}{N}\right]$ extends $A_{E_{1}}$.

2.7. Proposition. We assume that $A$ has compact factors. We have:

(a) the $E_{A \text {-schemes }} \mathcal{N}_{N E_{A}}$ and $\overline{\mathcal{N}}_{N E_{A}}$ coincide (i.e., $\mathcal{N}_{N E_{A}}=\overline{\mathcal{N}}_{N E_{A}}$ ); 
(b) if Theorem 1.2 holds for all abelian varieties over number fields which have compact factors, then the two equivalent statements of 2.6 hold.

Proof: As $\left(H_{A}, X_{A}\right)$ has compact factors, the $\mathbb{Q}$-rank of $H_{A}^{\text {ad }}$ is 0 . Thus the analytic space associated to $\operatorname{Sh}\left(H_{A}, X_{A}\right)_{\mathbb{C}} / K_{A}(N)$ is compact (see [2], Theorem 12.3 and Corollary 12.4). This implies that the analytic spaces associated to $\mathcal{N}_{N \mathbb{C}}$ and $\overline{\mathcal{N}}_{N \mathbb{C}}$ coincide. Thus $\mathcal{N}_{N \mathbb{C}}=\overline{\mathcal{N}}_{N \mathbb{C}}$. From this we get that (a) holds.

We prove (b). We show that the assumption that 2.6 (b) does not hold, leads to a contradiction. As $\mathcal{N}_{N E_{A}}=\overline{\mathcal{N}}_{N E_{A}}$ and as $\mathcal{N}_{N} \neq \overline{\mathcal{N}}_{N}$, we get that $\overline{\mathcal{N}}_{N}$ has points with values in finite fields which do not belong to $\mathcal{N}_{N}$. As the morphism $\overline{\mathcal{N}}_{N} \rightarrow \operatorname{Spec}\left(O_{E_{A}}\left[\frac{1}{N}\right]\right)$ is flat, it has quasi-sections in the quasi-finite, flat topology of $\operatorname{Spec}\left(O_{E_{A}}\left[\frac{1}{N}\right]\right)$ whose images contain any a priori given point of $\overline{\mathcal{N}}_{N}$ (cf. [13], Corollary (17.16.2)). From the last two sentences, we get that there exists a finite field extension $\tilde{E}$ of $E_{A}$ and a local $\operatorname{ring} \tilde{O}$ of $O_{\tilde{E}}$ of mixed characteristic such that we have a morphism $\tilde{u}: \operatorname{Spec}(\tilde{O}) \rightarrow \overline{\mathcal{N}}_{N}$ which does not factor through $\mathcal{N}_{N}$. Let $\tilde{A}$ be the generic fibre of $\tilde{u}^{*}(\overline{\mathcal{A}})$; it is an abelian variety over $\tilde{E}$. To reach a contradiction, we can assume that $E$ is a subfield of $\tilde{E}$ and that we have an embedding $i_{\tilde{E}}: \tilde{E} \hookrightarrow \mathbb{C}$ which extends $i_{E}$. Let $H_{\tilde{A}}$ be the Mumford-Tate group of $\tilde{A}_{\mathbb{C}}$. Let $H_{\tilde{t}}$ be an arbitrary simple factor of $H_{\tilde{A}}^{\text {ad }}$. As the generic fibre of $\tilde{u}$ factors through $\operatorname{Sh}\left(H_{A}, X_{A}\right) / K_{A}(N)$, the group $H_{\tilde{A}}$ is the Mumford-Tate group defined by a homomorphism $\mathbb{S} \rightarrow \mathrm{GL}_{W_{A}}$ which is an element of $X_{A}$. This implies that $\tilde{H}_{A}$ is naturally a subgroup of $H_{A}$. Thus we have natural inclusions $\operatorname{Lie}\left(H_{\tilde{t}}\right) \subseteq \operatorname{Lie}\left(H_{\tilde{A}}^{\mathrm{ad}}\right) \subseteq \operatorname{Lie}\left(H_{A}^{\mathrm{ad}}\right)$.

Let $H_{t}$ be a simple factor of $H_{A}^{\text {ad }}$ with the property that the natural Lie homomorphism $\operatorname{Lie}\left(H_{\tilde{t}}\right) \rightarrow \operatorname{Lie}\left(H_{t}\right)$ is a monomorphism of simple Lie algebras over $\mathbb{Q}$. Let $C_{t}$ be a simple, compact factor of $H_{t \mathbb{R}}$. Let $C_{\tilde{t}}$ be a simple factor of $H_{\tilde{t} \mathbb{R}}$ such that the simple Lie algebra Lie $\left(C_{\tilde{t}}\right)$ over $\mathbb{R}$ is naturally a Lie subalgebra of $\operatorname{Lie}\left(C_{t}\right)$. The group $C_{\tilde{t}}$ is isogenous to a subgroup of the compact group $C_{t}$ and thus it is compact. Thus the abelian variety $\tilde{A}$ has compact factors. Let $\tilde{E}_{1}$ be a finite field extension of $\tilde{E}$ such that $\tilde{A}_{\tilde{E}_{1}}$ extends to an abelian scheme over $O_{\tilde{E}_{1}}\left[\frac{1}{N}\right]$, cf. our hypotheses. Let $\tilde{v}_{1}$ be a prime of $\tilde{E}_{1}$ such that its local ring $\tilde{O}_{1}$ dominates $\tilde{O}$. As $\tilde{A}_{\tilde{E}_{1}}$ has good reduction with respect to $\tilde{v}_{1}$, the composite of the natural morphism $\operatorname{Spec}\left(\tilde{O}_{1}\right) \rightarrow \operatorname{Spec}(\tilde{O})$ with $\tilde{u}$, factors through $\mathcal{N}_{N}$ (cf. 2.5 (i)). Thus $\tilde{u}$ factors through $\mathcal{N}_{N}$. Contradiction. Thus 2.6 (b) holds. This proves (b).

\section{$\S 3$. A construction}

In this Section we assume that $A$ has compact factors (equivalently, that the Shimura pair $\left(H_{A}, X_{A}\right)$ has compact factors). For an abelian variety $B$ over $E$, let $\left(H_{B}, X_{B}\right)$ and $\left(H_{B}^{\mathrm{ad}}, X_{B}^{\mathrm{ad}}\right)$ be the analogues of $\left(H_{A}, X_{A}\right)$ and $\left(H_{A}^{\mathrm{ad}}, X_{A}^{\mathrm{ad}}\right)$ introduced in 2.4 but for $B$ instead of $A$. In this Section we will also assume that the adjoint group $H_{A}^{\text {ad }}$ is non-trivial. Let $\left(H_{0}, X_{0}\right)$ be a fixed simple factor of $\left(H_{A}^{\mathrm{ad}}, X_{A}^{\mathrm{ad}}\right)$. The homomorphism $h_{A 0}: \mathbb{S} \rightarrow H_{0 \mathbb{R}}$ of 2.4.1 is an element of $X_{0}$ and in fact $X_{0}$ is the $H_{0}(\mathbb{R})$-conjugacy class of $h_{A 0}$.

Let $p \in \mathbb{N}$ be a prime. In this Section we will prove the following result.

3.1. Theorem. Up to a replacement of $E$ by a finite field extension of it, there exists a principally polarized abelian variety $\left(A_{0}, \lambda_{A_{0}}\right)$ over $E$ such that the following two things hold: 
(a) we have a natural identity $\left(H_{A_{0}}^{\mathrm{ad}}, X_{A_{0}}^{\mathrm{ad}}\right)=\left(H_{0}, X_{0}\right)$ with the property that the homomorphism $h_{A_{0}}^{\text {ad }}: \mathbb{S} \rightarrow H_{A_{0} \mathbb{R}}^{\text {ad }}=H_{0 \mathbb{R}}$ is $h_{A 0}$;

(b) condition 2.6 (b) holds in the context of $\left(A_{0}, \lambda_{A_{0}}\right)$, for some number $N_{0} \in \mathbb{N} \backslash$ $\{1,2\}$ that is relatively prime to $p$.

The proof of this Theorem is carried out in 3.2 to 3.4. The existence (up to a replacement of $E$ by a finite field extension of it) of a principally polarized abelian variety $\left(A_{0}, \lambda_{A_{0}}\right)$ over $E$ such that (a) holds, is an elementary consequence of [7], Proposition 2.3.10. The hard part is to show that we can choose $\left(A_{0}, \lambda_{A_{0}}\right)$ so that (b) holds as well. In order to achieve that (b) holds, we will take $A_{0}$ so that the following two properties hold:

(i) the rank of the $\mathbb{Z}$-algebra $\operatorname{End}\left(A_{0 \mathbb{C}}\right)$ is very big;

(ii) if $A_{0}$ extends to a semiabelian scheme over a local ring of $O_{E}$ of mixed characteristic $(0, p)$, then the maximal torus of the special fibre of this semiabelian scheme extension, has a rank that is bounded from above only in terms of $\left(H_{0}, X_{0}\right)$.

It is easy to see that due to (i), the semiabelian scheme of (ii) is in fact an abelian scheme. Condition (b) will be implied by natural moduli analogues of (i) and (ii). In 3.2 we include notations that are essential for a review of the constructions of [7], Proposition 2.3.10 and for supplementing these constructions in order to be able to take $A_{0}$ so that the moduli analogues of (i) and (ii) hold. The mentioned review and supplementing process are the very essence of the construction of $A_{0}$ and are gathered in 3.3. In 3.4 we check that the moduli analogues of (i) and (ii) hold and we use this to end the proof of 3.1.

3.2. Notations. Let $F_{0}$ be a totally real number subfield of $\overline{\mathbb{Q}} \subseteq \mathbb{C}$ such that we have

$$
H_{0}=\operatorname{Res}_{F_{0} / \mathbb{Q}} G_{0},
$$

with $G_{0}$ as an absolutely simple adjoint group over $F_{0}$ (cf. [7], Subsubsection 2.3.4); the field $F_{0}$ is unique up to $\operatorname{Gal}(\mathbb{Q})$-conjugation. Let $T_{0}$ be a maximal torus of $H_{0}$. Let $B_{0}$ be a Borel subgroup of $H_{0 \overline{\mathbb{Q}}}$ that contains $T_{0 \overline{\mathbb{Q}}}$. Let $\mathfrak{D}_{0}$ be the Dynkin diagram of $\operatorname{Lie}\left(H_{0 \overline{\mathbb{Q}}}\right)$ with respect to $T_{0 \overline{\mathbb{Q}}}$ and $B_{0}$. We have $H_{0 \overline{\mathbb{Q}}}=\prod_{i \in \operatorname{Hom}\left(F_{0}, \mathbb{R}\right)} G_{0} \times_{F_{0}} i \overline{\mathbb{Q}}$. Thus $\mathfrak{D}_{0}$ is a disjoint union $\cup_{i \in \operatorname{Hom}\left(F_{0}, \mathbb{R}\right)} \mathfrak{D}_{i}$, where $\mathfrak{D}_{i}$ is the connected Dynkin diagram of $\operatorname{Lie}\left(G_{0} \times_{F_{0}} i \overline{\mathbb{Q}}\right)$. Let $\mathfrak{L}_{0}$ be the Lie type of a (any) simple factor of $H_{0 \mathbb{C}}$.

Let $\mathfrak{g}_{\mathfrak{n}}$ be the 1 dimensional Lie subalgebra of $\operatorname{Lie}\left(B_{0}\right)$ that corresponds to a node $\mathfrak{n}$ of $\mathfrak{D}_{0}$. The Galois group $\operatorname{Gal}(\mathbb{Q})$ acts on $\mathfrak{D}_{0}$ as follows. If $\gamma \in \operatorname{Gal}(\mathbb{Q})$, then $\gamma(\mathfrak{n})$ is the node of $\mathfrak{D}_{0}$ defined by the identity $\mathfrak{g}_{\gamma(\mathfrak{n})}=i_{g_{\gamma}}\left(\gamma\left(\mathfrak{g}_{\mathfrak{n}}\right)\right)$, where $i_{g_{\gamma}}$ is the inner conjugation of $\operatorname{Lie}\left(H_{0 \overline{\mathbb{Q}}}\right)$ by an element $g_{\gamma} \in H_{0}(\overline{\mathbb{Q}})$ which normalizes $T_{0 \overline{\mathbb{Q}}}$ and for which we have an identity $g_{\gamma} \gamma\left(B_{0}\right) g_{\gamma}^{-1}=B_{0}$.

Let $\mu_{0}: \mathbb{G}_{m \overline{\mathbb{Q}}} \rightarrow H_{0 \overline{\mathbb{Q}}}$ be as in 2.4.1. Let $\mathfrak{m}_{0}$ be the set of nodes of $\mathfrak{D}_{0}$ such that the unique cocharacter of $T_{0 \overline{\mathbb{Q}}}$ that acts on $\mathfrak{g}_{\mathfrak{n}}$ trivially if $\mathfrak{n} \notin \mathfrak{m}_{0}$ and via the identical character of $\mathbb{G}_{m \overline{\mathbb{Q}}}$ if $\mathfrak{n} \in \mathfrak{m}_{0}$, is $H_{0}(\overline{\mathbb{Q}})$-conjugate to $\mu_{0}$. Let $\mathfrak{o}_{0}$ be the set of nodes of $\mathfrak{D}_{0}$ formed by the orbit of $\mathfrak{m}_{0}$ under $\operatorname{Gal}(\mathbb{Q})$. As $H_{A}$ is the smallest subgroup of $\mathrm{GL}_{W_{A}}$ through which $h_{A}$ factors, the images of both $h_{A 0}$ and $\mu_{0}$ are non-trivial. Thus the set $\mathfrak{m}_{0}$ is non-empty. The image of $h_{A 0}$ in a simple factor $\mathcal{F}_{0}$ of $H_{0 \mathbb{R}}$ is trivial if and only if the group $\mathcal{F}_{0}$ is compact 
(this is so as the centralizer of $\operatorname{Im}\left(h_{A}^{\text {ad }}\right)$ in $H_{A \mathbb{R}}^{\text {ad }}$ is a maximal compact subgroup of $H_{A \mathbb{R}}^{\text {ad }}$, cf. [7], page 259). As $H_{0 \mathbb{R}}$ has at least one simple, compact factor (cf. def. 1.1), we get that:

- there exists an element $i_{0} \in \operatorname{Hom}\left(F_{0}, \mathbb{R}\right)$ such that $\mathfrak{m}_{0}$ contains no node of $\mathfrak{D}_{i_{0}}$ (equivalently, such that the simple factor $G_{0} \times_{F_{0}} i_{0} \mathbb{R}$ of $H_{0 \mathbb{R}}$ is compact).

As the Hodge $\mathbb{Q}$-structure on $\operatorname{Lie}\left(H_{0}\right)$ defined by any element $x_{0} \in X_{0}$ is of type $\{(-1,1),(0,0),(1,-1)\}$, for each $i \in \operatorname{Hom}\left(F_{0}, \mathbb{R}\right)$ the set $\mathfrak{m}_{0}$ contains at most one node of $\mathfrak{D}_{i}$. We know that $\mathfrak{L}_{0}$ is a classical Lie type, cf. [7], Table 2.3.8. Moreover, if $\mathfrak{m}_{0}$ contains a node of $\mathfrak{D}_{i}$, then with the standard notations of [4], Plates I to VI, this node is (cf. [7], Table 1.3.9): an arbitrary node if $\mathfrak{L}_{0}=A_{n}$, node 1 if $\mathfrak{L}_{0}=B_{n}$, node $n$ if $\mathfrak{L}_{0}=C_{n}$, and an extremal node if $\mathfrak{L}_{0}=D_{n}$. The reflex field $E\left(H_{0}, X_{0}\right)$ of $\left(H_{0}, X_{0}\right)$ is the fixed field of the open subgroup of $\operatorname{Gal}(\mathbb{Q})$ that stabilizes $\mathfrak{m}_{0}$, cf. [7], Proposition 2.3.6.

If the Lie type $\mathfrak{L}_{0}$ is $A_{n}, B_{n}$, or $C_{n}$, then $\left(H_{0}, X_{0}\right)$ is said to be of $A_{n}, B_{n}$, or $C_{n}$ type. If $\mathfrak{L}_{0}=D_{n}$ with $n \geq 5$, then $\left(H_{0}, X_{0}\right)$ is said to be:

- of $D_{n}^{\mathbb{R}}$ type, if for each embedding $i: F_{0} \hookrightarrow \mathbb{R}, \mathfrak{o}_{0}$ contains only the node 1 of $\mathfrak{D}_{i}$;

- of $D_{n}^{\mathbb{H}}$ type, if for each embedding $i: F_{0} \hookrightarrow \mathbb{R}, \mathfrak{o}_{0}$ contains either only node $n-1$ of $\mathfrak{D}_{i}$, or only the node $n$ of $\mathfrak{D}_{i}$, or only the nodes $n-1$ and $n$ of $\mathfrak{D}_{i}$.

If $\mathfrak{L}_{0}=D_{4}$, then $\left(H_{0}, X_{0}\right)$ is said to be of $D_{4}^{\mathbb{R}}$ (resp. of $\left.D_{4}^{\mathbb{H}}\right)$ if for each embedding $i: F_{0} \hookrightarrow \mathbb{R}, \mathfrak{o}_{0}$ contains only one (resp. exactly two) nodes of $\mathfrak{D}_{i}$; with the notations of [4], Plate IV, this node (resp. these two nodes) will be chosen in what follows to be the node 1 (resp. to be the last two nodes 3 and 4 ).

The definition of the $A_{n}, B_{n}, C_{n}, D_{n}^{\mathbb{H}}$, and $D_{n}^{\mathbb{R}}$ types conforms with [7]. From [7], Table 2.3.8 we get that $\left(H_{0}, X_{0}\right)$ is of $A_{n}, B_{n}, C_{n}, D_{n}^{\mathbb{H}}$, or $D_{n}^{\mathbb{R}}$ type.

3.2.1. Enlarging $F_{0}$. For a totally real number subfield $F_{1}$ of $\overline{\mathbb{Q}}$ that contains $F_{0}$, let

$$
G_{1}:=G_{0 F_{1}} \text { and } H_{1}:=\operatorname{Res}_{F_{1} / \mathbb{Q}} G_{1}
$$

and let $i_{\text {nat }}: F_{1} \hookrightarrow \mathbb{R}$ be the natural embedding. We have a natural monomorphism $G_{0} \hookrightarrow \operatorname{Res}_{F_{1} / F_{0}} G_{1}$ over $F_{0}$. The $\operatorname{Res}_{F_{0} / \mathbb{Q}}$ of this last monomorphism is a monomorphism $H_{0}=\operatorname{Res}_{F_{0} / \mathbb{Q}} G_{0} \hookrightarrow H_{1}=\operatorname{Res}_{F_{1} / \mathbb{Q}} G_{1}$ that extends to an injective map of Shimura pairs

$$
\left(H_{0}, X_{0}\right) \hookrightarrow\left(H_{1}, X_{1}\right) .
$$

Let $h_{A 1}: \mathbb{S} \rightarrow H_{1 \mathbb{R}}$ be the homomorphism that is the composite of $h_{A 0}$ with the natural monomorphism $H_{0 \mathbb{R}} \hookrightarrow H_{1 \mathbb{R}}$. Thus $h_{A 1} \in X_{1}$ and in fact $X_{1}$ is the $H_{1}(\mathbb{R})$-conjugacy class of $h_{A 1}$. As $H_{1 \mathbb{R}} \simeq H_{0 \mathbb{R}}^{\left[F_{1}: F_{0}\right]}$, the hermitian symmetric domain $X_{1}$ is isomorphic to $X_{0}^{\left[F_{1}: F_{0}\right]}$.

The centralizer of $T_{0}$ in $H_{1}$ is a maximal torus $T_{1}$ of $H_{1}$. Let $B_{1}$ be the unique Borel subgroup of $H_{1 \overline{\mathbb{Q}}}$ that contains both $T_{1 \overline{\mathbb{Q}}}$ and $B_{0}$. Let $\left(\mathfrak{D}_{1}, \mathfrak{m}_{1}, \mathfrak{o}_{1}\right)$ be the analogue of the triple $\left(\mathfrak{D}_{0}, \mathfrak{m}_{0}, \mathfrak{o}_{0}\right)$ but associated to the Shimura pair $\left(H_{1}, X_{1}\right)$ and to the pair $\left(T_{1 \overline{\mathbb{Q}}}, B_{1}\right)$.

Let $s: \mathfrak{D}_{1} \rightarrow \mathfrak{D}_{0}$ be the $\operatorname{Gal}(\mathbb{Q})$-invariant surjective map of Dynkin diagrams which is naturally associated to the monomorphism $H_{0 \overline{\mathbb{Q}}} \hookrightarrow H_{1 \overline{\mathbb{Q}}} \stackrel{\sim}{\rightarrow} H_{0 \overline{\mathbb{Q}}}^{\left[F_{1}: F_{0}\right]}$; if $i: F_{0} \hookrightarrow \mathbb{R}$ is an arbitrary embedding, then $s^{-1}\left(\mathfrak{D}_{i}\right)$ is the disjoint union of the connected Dynkin diagrams 
of the simple factors of $\operatorname{Lie}\left(H_{1 \overline{\mathbb{Q}}}\right)$ that correspond to those elements of $\operatorname{Hom}\left(F_{1}, \mathbb{R}\right)$ which extend $i: F_{0} \hookrightarrow \mathbb{R}$. For simplicity, in what follows we will also denote by $i$ an arbitrary element of $\operatorname{Hom}\left(F_{1}, \mathbb{R}\right)$. We have identities $s^{-1}\left(\mathfrak{m}_{0}\right)=\mathfrak{m}_{1}$ and $s^{-1}\left(\mathfrak{o}_{0}\right)=\mathfrak{o}_{1}$. The existence of the map $s$ and the identity $s^{-1}\left(\mathfrak{o}_{0}\right)=\mathfrak{o}_{1}$ imply that the types of $\left(H_{0}, X_{0}\right)$ and $\left(H_{1}, X_{1}\right)$ are equal. As $H_{1 \mathbb{R}} \underset{\rightarrow}{\rightarrow} H_{0 \mathbb{R}}^{\left[F_{1}: F_{0}\right]}$ and as $H_{0 \mathbb{R}}$ has at least one simple, compact factor, the simple, adjoint Shimura pair $\left(H_{1}, X_{1}\right)$ has compact factors.

Let $\mathfrak{S}_{1}$ be the subset of nodes of $\mathfrak{D}_{1}$ defined as follows:

- if $\left(H_{0}, X_{0}\right)$ is of $A_{n}$ type, then $\mathfrak{S}_{1}$ is the set of all extremal nodes;

- if $\left(H_{0}, X_{0}\right)$ is of $B_{n}$ (resp. $\left.C_{n}\right)$ type, then $\mathfrak{S}_{1}$ is the set of all nodes $n$ (resp. 1);

- if $\left(H_{0}, X_{0}\right)$ is of $D_{n}^{\mathbb{H}}$ (resp. $\left.D_{n}^{\mathbb{R}}\right)$ type, then $\mathfrak{S}_{1}$ is the set of all nodes 1 (resp. $n-1$ and $n)$.

The set $\mathfrak{S}_{1}$ is $\operatorname{Gal}\left(\mathbb{Q}\right.$ )-invariant (if $\mathfrak{L}_{0}=D_{n}$, this is implied by the very definitions of the $D_{n}^{\mathbb{H}}$ and $D_{n}^{\mathbb{R}}$ types). We can identify the $\operatorname{Gal}(\mathbb{Q})$-set $\mathfrak{S}_{1}$ with $\operatorname{Hom}\left(\tilde{F}_{1}, \mathbb{C}\right)$ for an étale $F_{1}$-algebra of degree at most 2 . The degree of $\tilde{F}_{1}$ is 2 if and only if $\left(H_{0}, X_{0}\right)$ is either of $A_{n}$ type with $n \geq 2$ or of $D_{n}^{\mathbb{R}}$ type with $n \geq 4$. The $F_{1}$-algebra $\tilde{F}_{1}$ is either a field of CM type (cf. [7], Subsubsection 2.3.4 (b) or the first paragraph of the proof of Proposition 2.3.10) or a product of two fields isomorphic to $F_{1}$ and thus of CM type. We emphasize that if $\left[\tilde{F}_{1}: F_{1}\right]=2$ and $\left(H_{0}, X_{0}\right)$ is of $D_{n}^{\mathbb{R}}$ type with $n$ even, then $\tilde{F}_{1}$ is not necessarily a field.

3.2.2. Choice of $F_{1}$. Let $\overline{\mathbb{Q}_{p}}$ be an algebraic closure of $\mathbb{Q}_{p}$. We fix an identification between $\overline{\mathbb{Q}}$ an the algebraic closure of $\mathbb{Q}$ in $\overline{\mathbb{Q}_{p}}$ and we use it to identify naturally the set $\operatorname{Hom}\left(F_{0}, \mathbb{R}\right)=\operatorname{Hom}\left(F_{0}, \overline{\mathbb{Q}}\right)$ with $\operatorname{Hom}\left(F_{0}, \overline{\mathbb{Q}} p\right)$. We write $F_{0} \otimes_{\mathbb{Q}} \mathbb{Q}_{p}=\prod_{j \in J} F_{0 j}$ as a product of $p$-adic fields. Let $j_{0} \in J$ be the unique element with the property that to the embedding $i_{0}: F_{0} \hookrightarrow \mathbb{R}$ corresponds (under the identification $\operatorname{Hom}\left(F_{0}, \mathbb{R}\right)=\operatorname{Hom}\left(F_{0}, \overline{\mathbb{Q}_{p}}\right)$ ) an embedding $F_{0} \hookrightarrow \overline{\mathbb{Q}_{p}}$ that factors through $F_{0 j_{0}}$. We will choose the field $F_{1}$ such that $\left[F_{1}: F_{0}\right]>>0$ and moreover the following property holds:

$(*)$ we have a natural product decomposition $F_{1} \otimes_{\mathbb{Q}} \mathbb{Q}_{p}=\prod_{j \in J} F_{1 j}$ into $p$-adic fields, where $F_{1 j}=F_{0 j}$ if $j \in J \backslash\left\{j_{0}\right\}$ and where $F_{1 j_{0}}$ is a finite field extension of $F_{0 j_{0}}$.

3.2.3. Choice of $K_{1}$. Let $v_{1 j_{0}}$ be the prime of $F_{1}$ such that the completion of $F_{1}$ with respect to $v_{1 j_{0}}$ is the factor $F_{1 j_{0}}$ of $F_{1} \otimes_{\mathbb{Q}} \mathbb{Q}_{p}$. Let $K_{1}$ be a totally imaginary quadratic extension of $F_{1}$ which is unramified above primes of $F_{1}$ that divide $p$ and which has only one prime $w_{1 j_{0}}$ above $v_{1 j_{0}}$. Let $K_{1 j_{0}}$ be the completion of $K_{1}$ with respect to $w_{1 j_{0}}$; we have $\left[K_{1 j_{0}}: F_{1 j_{0}}\right]=2$. As $F_{1}$ is a totally real number field and as $K_{1}$ is a totally imaginary quadratic extension of $F_{1}$, the field $K_{1}$ is of CM type.

3.3. Lemma. There exists a Shimura pair $\left(H_{2}, X_{2}\right)$ such that the following five things hold:

(i) the adjoint Shimura pair of $\left(H_{2}, X_{2}\right)$ is $\left(H_{1}, X_{1}\right)$ and there exists $h_{A_{0}} \in X_{2}$ that maps naturally into the element $h_{A 1} \in X_{1}$ introduced in 3.2.1;

(ii) we have an injective map $f_{2}:\left(H_{2}, X_{2}\right) \hookrightarrow\left(G S p\left(W_{2}, \psi_{2}\right), S_{2}\right)$ into a Shimura pair that defines a Siegel modular variety; 
(iii) the torus $\mathcal{T}:=\operatorname{Res}_{K_{1} \otimes_{F_{1}} \tilde{F}_{1} / \mathbb{Q}} \mathbb{G}_{m K_{1} \otimes_{F_{1}} \tilde{F}_{1}}$ is naturally a subgroup of $G L_{W_{2}}$ that centralizes $H_{2}^{\text {der }}$ and that makes $W_{2}$ to have a natural structure of a $K_{1}$-vector space;

(iv) the torus $Z^{0}\left(H_{2}\right)$ is the torus of $G L_{W_{2}}$ generated by $Z\left(G L_{W_{2}}\right)$ and by the maximal subtorus $\mathcal{T}_{c}$ of $\mathcal{T}$ which over $\mathbb{R}$ is compact;

(v) if the direct sum decomposition $W_{2} \otimes_{\mathbb{Q}} \mathbb{Q}_{p}=\oplus_{j \in J} W_{2 j}$ into $F_{1} \otimes_{\mathbb{Q}} \mathbb{Q}_{p}$-modules has the property that each $W_{2 j}$ is an $F_{1 j}$-vector space, then there exists $d_{0} \in \mathbb{N}$ that depends only on $\left(H_{0}, X_{0}\right)$ (and not on $F_{1}$ ) and such that we have an inequality $\sum_{j \in J \backslash\left\{j_{0}\right\}} \operatorname{dim}_{\mathbb{Q}_{p}}\left(W_{2 j}\right) \leq d_{0}$.

Proof: The existence of the Shimura pair $\left(H_{2}, X_{2}\right)$ such that (i) and (ii) (resp. (iii) to (v)) hold follows from the statement (resp. from the proof) of [7], Proposition 2.3.10. We now recall the details of the construction of $\left(H_{2}, X_{2}\right)$, in the form needed in what follows. Let $Q_{0}$ be a maximal torus of $G_{0}$. Its rank $n$ is equal to the rank of $\mathfrak{L}_{0}$. To ease the notations, we can assume that we have an identity $T_{0}=\operatorname{Res}_{F_{0} / \mathbb{Q}} Q_{0}$ between tori of $H_{0}$.

Let $E_{0}$ be the smallest number field which contains $F_{0}$ and which has the property that the torus $Q_{0 E_{0}}$ is split; it is a Galois extension of $F_{0}$ and the Galois group $\operatorname{Gal}\left(E_{0} / F_{0}\right)$ is a finite subgroup of $\mathrm{GL}_{X_{*}\left(Q_{0 E_{0}}\right)}(\mathbb{Z})$, where the group of cocharacters of $X_{*}\left(Q_{0 E_{0}}\right)$ of $Q_{0 E_{0}}$ is viewed as a free $\mathbb{Z}$-module of rank $n$. Let $E_{1}$ be the composite field of $E_{0}$ and $F_{1}$. As the finite subgroups of $\mathrm{GL}_{X_{*}\left(Q_{0 E_{0}}\right)}(\mathbb{Z})$ have orders bounded from above only in terms of $n$, the degree $\left[E_{0}: F_{0}\right]$ is bounded from above only in terms of $n$. Thus the degree $\left[E_{1}: F_{1}\right]$ is bounded from above by a number $d_{1} \in \mathbb{N}$ that depends only on $\left(H_{0}, X_{0}\right)$.

We will consider a representation $\rho_{1}: G_{1 E_{1}}^{\mathrm{sc}} \rightarrow \mathrm{GL}_{V_{1}}$, where $V_{1}$ is an $E_{1}$-vector space of finite dimension. In what follows all the weights used are with respect to the maximal torus of $G_{1 E_{1}}^{\mathrm{sc}}$ whose image in $G_{1 E_{1}}$ is the maximal torus $Q_{0 E_{1}}$ of $G_{0 E_{1}}=G_{1 E_{1}}$. Depending on the type of $\left(H_{0}, X_{0}\right)$, we choose $\rho_{1}$ such that (cf. the definition of the subset $\mathfrak{S}_{1}$ of nodes of $\mathfrak{D}_{1}$ ) the following things hold (see [4], Plates I to IV for the weights used):

(via) if $\left(H_{0}, X_{0}\right)$ is of $A_{n}$ type with $n \geq 2$, then $\rho_{1}$ is the direct sum of the faithful representations of $G_{1 E_{1}}^{\mathrm{sc}}=\mathrm{SL}_{n+1 E_{1}}$ associated to the weights $\varpi_{1}$ and $\varpi_{n}$ (thus $\operatorname{dim}_{E_{1}}\left(V_{1}\right)=$ $2 n+2)$;

(vib) if $\left(H_{0}, X_{0}\right)$ is of $B_{n}$ type with $n \geq 3$, then $\rho_{1}$ is the faithful spin representation of $G_{1 E_{1}}^{\mathrm{sc}}=\operatorname{Spin}_{2 n+1 E_{1}}$ associated to the weight $\varpi_{n}\left(\right.$ thus $\left.\operatorname{dim}_{E_{1}}\left(V_{1}\right)=2^{n}\right)$;

(vic) if $\left(H_{0}, X_{0}\right)$ is of $C_{n}$ type with $n \geq 1$, then $\rho_{1}$ is the faithful representation of $G_{1 E_{1}}^{\mathrm{sc}}=\operatorname{Sp}_{2 n E_{1}}$ of dimension $2 n$ associated to the weight $\varpi_{1}$ (thus $\operatorname{dim}_{E_{1}}\left(V_{1}\right)=2 n$ );

(vid1) if $\left(H_{0}, X_{0}\right)$ is of $D_{n}^{\mathbb{H}}$ type with $n \geq 4$, then $\rho_{1}$ is the representation of $G_{1 E_{1}}^{\mathrm{sc}}$ of dimension $2 n$ associated to the weight $\varpi_{1}\left(\right.$ thus $\left.\operatorname{dim}_{E_{1}}\left(V_{1}\right)=2 n\right)$;

(vid2) if $\left(H_{0}, X_{0}\right)$ is of $D_{n}^{\mathbb{R}}$ type with $n \geq 4$, then $\rho_{1}$ is the spin representation of $G_{1 E_{1}}^{\mathrm{sc}}=\operatorname{Spin}_{2 n E_{1}}$ associated to the weights $\varpi_{n-1}$ and $\varpi_{n}$ (thus $\operatorname{dim}_{E_{1}}\left(V_{1}\right)=2^{n}$ ).

Let $V$ be $V_{1}$ but viewed as a rational vector space; we keep in mind that $V$ has also a natural structure of an $E_{1}$-vector space and so also of an $F_{1}$-vector space. As $H_{1}^{\mathrm{sc}}=\operatorname{Res}_{F_{1} / \mathbb{Q}} G_{1}^{\mathrm{sc}}$ is a subgroup of $\operatorname{Res}_{E_{1} / \mathbb{Q}} G_{1 E_{1}}^{\mathrm{sc}}, V$ is naturally an $H_{1}^{\mathrm{sc}}$-module. Let $H_{2}^{\text {der }}$ be the image of the natural representation $H_{1}^{\mathrm{sc}} \rightarrow \mathrm{GL}_{V}$ over $\mathbb{Q}$; the adjoint group of $H_{2}^{\text {der }}$ is $H_{1}$. The set of weights used in (via) to (vid2) is stable under the natural action of $\operatorname{Gal}\left(F_{1}\right)$ on the abelian group of weights and as a $\operatorname{Gal}\left(F_{1}\right)$-set it can be identified with the 
$\operatorname{Gal}\left(F_{1}\right)$-set of nodes of $\mathfrak{D}_{i_{\text {nat }}}$ contained in $\mathfrak{S}_{1}$. This implies that the center of the double centralizer of $H_{2}^{\text {der }}$ in $\mathrm{GL}_{V}$ is the torus $\operatorname{Res}_{\tilde{F}_{1} / \mathbb{Q}} \mathbb{G}_{m \tilde{F}_{1}}$ (see 3.2 .1 for $\tilde{F}_{1}$ ).

We take $W_{2}:=K_{1} \otimes_{F_{1}} V$ and we view it as a rational vector space. As $W_{2}$ has also a natural structure of a $K_{1} \otimes_{F_{1}} \tilde{F}_{1}$-module whose annihilator is trivial, the torus $\mathcal{T}:=\operatorname{Res}_{K_{1} \otimes_{F_{1}} \tilde{F}_{1} / \mathbb{Q}} \mathbb{G}_{m K_{1} \otimes_{F_{1}} \tilde{F}_{1}}$ is naturally a subgroup of $\mathrm{GL}_{W_{2}}$. Moreover $W_{2}$ has a natural structure of a $K_{1}$-vector space. We will also identify $H_{2}^{\text {der }}$ with a semisimple subgroup of $\mathrm{GL}_{W_{2}}$ that commutes with $\mathcal{T}$. As $K_{1}$ and the simple factors of $\tilde{F}_{1}$ are fields of CM type (cf. 3.2.1 and 3.2.3), the maximal compact subtorus of $\mathcal{T}_{\mathbb{R}}$ is the extension to $\mathbb{R}$ of a subtorus $\mathcal{T}_{c}$ of $\mathcal{T}$. Let $Z^{0}\left(H_{2}\right)$ be the torus of $\mathrm{GL}_{W_{2}}$ generated by $\mathcal{T}_{c}$ and $Z\left(\mathrm{GL}_{W_{2}}\right)$. The torus $Z^{0}\left(H_{2}\right)$ commutes with $H_{2}^{\text {der }}$ and therefore there exists a reductive subgroup $H_{2}$ of $\mathrm{GL}_{W_{2}}$ such that the notations match (i.e., the derived group of $H_{2}$ is $H_{2}^{\text {der }}$ and the maximal torus of the center of $H_{2}$ is $Z^{0}\left(H_{2}\right)$ ). Thus (iv) holds. As $H_{2}$ commutes with $\mathcal{T}$, (iii) also holds. Let $H_{3}$ be the subgroup of $\mathrm{GL}_{W_{2}}$ generated by $H_{2}^{\text {der }}$ and $\mathcal{T}$; it contains $H_{2}$.

The existence of an injective map $f_{2}:\left(H_{2}, X_{2}\right) \hookrightarrow\left(\operatorname{GSp}\left(W_{2}, \psi_{2}\right), S_{2}\right)$ such that (i) holds is part of the proof of [7], Proposition 2.3.10. We recall the part of loc. cit. that pertains to the existence of $h_{A_{0}}$. We have $F_{1} \otimes_{\mathbb{Q}} \mathbb{R}=\prod_{i \in \operatorname{Hom}\left(F_{1}, \mathbb{R}\right)} \mathbb{R} ;$ for $i \in \operatorname{Hom}\left(F_{1}, \mathbb{R}\right)$ let $\pi_{i}$ be the idempotent of $F_{1} \otimes_{\mathbb{Q}} \mathbb{R}$ such that $\pi_{i}\left(F_{1} \otimes_{\mathbb{Q}} \mathbb{R}\right)$ is the factor $\mathbb{R}$ of $F_{1} \otimes_{\mathbb{Q}} \mathbb{R}$ that corresponds to $i$. Let $V(i):=\pi_{i} W_{2} \otimes_{\mathbb{Q}} \mathbb{R}$. We have a direct sum decomposition $W_{2} \otimes \mathbb{Q} \mathbb{R}=\oplus_{i \in \operatorname{Hom}\left(F_{1}, \mathbb{R}\right)} V(i)$ of $H_{2 \mathbb{R}}^{\text {der }}$-modules. We also have a direct sum decomposition

$$
W_{2} \otimes_{\mathbb{Q}} \mathbb{C}=\oplus_{i \in \operatorname{Hom}\left(F_{1}, \mathbb{R}\right)} W\left(i_{1}\right) \oplus W\left(i_{2}\right)
$$

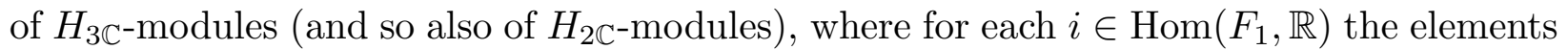
$i_{1}, i_{2} \in \operatorname{Hom}\left(K_{1}, \mathbb{C}\right)$ extend $i \in \operatorname{Hom}\left(F_{1}, \mathbb{C}\right)$ and are listed in an a priori chosen order and where $V(i) \otimes_{\mathbb{R}} \mathbb{C}=W\left(i_{1}\right) \oplus W\left(i_{2}\right)$ is the natural decomposition into $K_{1} \otimes_{F_{1}} i \mathbb{R} \otimes_{\mathbb{R}} \mathbb{C}$ modules. Any homomorphism $h_{A_{0}}: \mathbb{S} \rightarrow H_{3 \mathbb{R}}$ normalizes $V(i)$ and thus gives birth to a homomorphism $h_{A_{0}, i}: \mathbb{S} \rightarrow \mathrm{GL}_{V(i)}$. Moreover, any homomorphism $h_{A_{0}}: \mathbb{S} \rightarrow H_{3 \mathbb{R}}$ that defines a Hodge $\mathbb{Q}$-structure on $W_{2}$ which has a (constant) weight, factors through $H_{2 \mathbb{R}}$.

We will choose a homomorphism $h_{A_{0}}: \mathbb{S} \rightarrow H_{3 \mathbb{R}}$ such that the Hodge $\mathbb{Q}$-structure on $W_{2}$ is of type $\{(-1,0),(0,-1)\}$ (i.e., we have a natural Hodge decomposition $W_{2} \otimes_{\mathbb{Q}} \mathbb{C}=$ $F_{A_{0}}^{-1,0} \oplus F_{A_{0}}^{0,-1}$ defined by $\left.h_{A_{0}}\right)$ and the following two additional properties hold:

(viic) if $i \in \operatorname{Hom}\left(F_{1}, \mathbb{R}\right)$ is such that $G_{1} \times_{F_{1}} i \mathbb{R}$ is compact (for instance, if $i \in$ $\operatorname{Hom}\left(F_{1}, \mathbb{R}\right)$ extends $\left.i_{0} \in \operatorname{Hom}\left(F_{0}, \mathbb{R}\right)\right)$, then $h_{A_{0}, i}$ is fixed by the image of $H_{3 \mathbb{R}}$ in $\mathrm{GL}_{V(i)}$, we have $W\left(i_{1}\right) \subseteq F_{A_{0}}^{-1,0}$ and $W\left(i_{2}\right) \subseteq F_{A_{0}}^{0,-1}$, and thus $W\left(i_{1}\right)^{*}$ is included in the Hodge filtration $F_{A_{0}}^{1}\left(W_{2}^{*} \otimes_{\mathbb{Q}} \mathbb{C}\right)$ of $W_{2}^{*} \otimes_{\mathbb{Q}} \mathbb{C}$ defined by $h_{A_{0}} ;$

(viin) if $i \in \operatorname{Hom}\left(F_{1}, \mathbb{R}\right)$ is such that $G_{1} \times_{F_{1}} i \mathbb{R}$ is non-compact, then $h_{A_{0}, i}: \mathbb{S} \rightarrow$ $\mathrm{GL}_{V(i)}$ is the unique homomorphism such that the Hodge $\mathbb{R}$-structure on $V(i)$ is of type $\{(-1,0),(0,-1)\}$ and $h_{A_{0}, i}$ lifts the non-trivial homomorphism $\mathbb{S} \rightarrow G_{1} \times_{F_{1}} i \mathbb{R}$ defined naturally by $h_{A 1}$ (here $G_{1} \times_{F_{1}} i \mathbb{R}$ is a simple factor of $\left.H_{1 \mathbb{R}}=\prod_{\tilde{i} \in \operatorname{Hom}\left(F_{1}, \mathbb{R}\right)} G_{1} \times_{F_{1} \tilde{i}} \mathbb{R}\right)$.

See proof of [7], Proposition 2.3.10 for the explicit construction of $h_{A_{0}, i}$ of (viin); below we will only use (viic). We denote also by $h_{A_{0}}: \mathbb{S} \rightarrow H_{2 \mathbb{R}}$ the factorization of $h_{A_{0}}$ through $H_{2 \mathbb{R}}$ (the weight of the Hodge $\mathbb{Q}$-structure on $W_{2}$ defined by $h_{A_{0}}$ is -1 ). Let $X_{2}$ be the $H_{2}\left(\mathbb{R}\right.$ )-conjugacy class of $h_{A_{0}}: \mathbb{S} \rightarrow H_{2 \mathbb{R}}$. From (viic) and (viin) we get that (i) 
holds. The existence of an injective map as in (ii) is a particular case of the argument for [7], Corollary 2.3.3. Thus (i) and (ii) hold. From (viic) we get that:

(viii) for each $i \in \operatorname{Hom}\left(F_{1}, \mathbb{R}\right)$ that extends $i_{0} \in \operatorname{Hom}\left(F_{0}, \mathbb{R}\right)$ and each $x_{2} \in X_{2}$, $W\left(i_{1}\right)^{*}$ is included in the Hodge filtration $F_{x_{2}}^{1}\left(W_{2}^{*} \otimes_{\mathbb{Q}} \mathbb{C}\right)$ of $W_{2}^{*} \otimes_{\mathbb{Q}} \mathbb{C}$ defined by $x_{2}$.

We check that $(\mathrm{v})$ also holds. Let $j \in J \backslash\left\{j_{0}\right\}$. We have $\operatorname{dim}_{E_{1}}\left(V_{1}\right) \leq 2^{n+1}$ (cf. (via) to $(\operatorname{vid} 2)),\left[F_{1 j}: \mathbb{Q}_{p}\right]=\left[F_{0 j}: \mathbb{Q}_{p}\right] \leq\left[F_{0}: \mathbb{Q}\right]$ (cf. 3.2.2(*)), and $\operatorname{dim}_{F_{1 j}}\left(E_{1} \otimes_{F_{1}} F_{1 j}\right) \leq d_{1}$. As $W_{2} \otimes_{\mathbb{Q}} \mathbb{Q}_{p}=K_{1} \otimes_{F_{1}} V \otimes_{\mathbb{Q}} \mathbb{Q}_{p}=K_{1} \otimes_{F_{1}} V_{1} \otimes_{E_{1}} E_{1} \otimes_{F_{1}} F_{1} \otimes_{\mathbb{Q}} \mathbb{Q}_{p}$, we can identify $W_{2 j}$ with $K_{1} \otimes_{F_{1}} V_{1} \otimes_{E_{1}} E_{1} \otimes_{F_{1}} F_{1 j}$. Thus

$$
\begin{gathered}
\operatorname{dim}_{\mathbb{Q}_{p}}\left(W_{2 j}\right)=\left[K_{1}: F_{1}\right] \operatorname{dim}_{E_{1}}\left(V_{1}\right) \operatorname{dim}_{\mathbb{Q}_{p}}\left(E_{1} \otimes_{F_{1}} F_{1 j}\right) \leq 22^{n+1} \operatorname{dim}_{\mathbb{Q}_{p}}\left(E_{1} \otimes_{F_{1}} F_{1 j}\right)= \\
=2^{n+2} \operatorname{dim}_{F_{1 j}}\left(E_{1} \otimes_{F_{1}} F_{1 j}\right)\left[F_{1 j}: \mathbb{Q}_{p}\right] \leq 2^{n+2} d_{1}\left[F_{1 j}: \mathbb{Q}_{p}\right] \leq 2^{n+2} d_{1}\left[F_{0}: \mathbb{Q}\right] .
\end{gathered}
$$

Thus $\operatorname{dim}_{\mathbb{Q}_{p}}\left(W_{2 j}\right)$ is bounded from above only in terms of $\left(H_{0}, X_{0}\right)$. As the cardinality $|J|$ of the set $J$ depends only on $F_{0}$, we get that there exists $d_{0} \in \mathbb{N}$ that depends only on $\left(H_{0}, X_{0}\right)$ (and not on $F_{1}$ ) and such that we have $\sum_{j \in J \backslash\left\{j_{0}\right\}} \operatorname{dim}_{\mathbb{Q}_{p}}\left(W_{2 j}\right) \leq d_{0}$. For instance, we can take $d_{0}$ to be $2^{n+2} d_{1}(|J|-1)\left[F_{0}: \mathbb{Q}\right]$. Thus $(\mathrm{v})$ also holds.

3.4. The proof of 3.1. We will take $F_{1}$ such that besides $3.2 .2\left(^{*}\right)$ we also have an inequality $\left[F_{1}: \mathbb{Q}\right]>\frac{d_{0}}{2}$; this inequality will later on turn out to be the moduli analogue of 3.1 (i). Let $H_{A_{0}}$ be the smallest subgroup of $H_{2}$ such that the homomorphism $h_{A_{0}}: \mathbb{S} \rightarrow H_{2 \mathbb{R}}$ of 3.3 (i) factors through $H_{A_{0} \mathbb{R}}$. As either $2 \pi i \psi_{2}$ or $-2 \pi i \psi_{2}$ is a polarization of the Hodge $\mathbb{Q}$-structure on $W_{2}$ defined by $h_{A_{0}}$ (cf. 3.3 (ii)), the group $H_{A_{0}}$ is reductive (cf. [8], Proposition 3.6). As $h_{A 1} \in X_{1}$ is the image of $h_{A 0} \in X_{0}$, we have a natural identity $H_{A_{0}}^{\text {ad }}=H_{0}$. Let $X_{A_{0}}$ be the $H_{A_{0}}(\mathbb{R})$-conjugacy class of $h_{A_{0}}: \mathbb{S} \rightarrow H_{A_{0} \mathbb{R}}$. Let $L_{A_{0}}$ be a $\mathbb{Z}$-lattice of $W_{2}$ such that $\psi_{2}$ induces a perfect alternating form on $L_{A_{0}}$.

We have an injective map $f_{A_{0}}:\left(H_{A_{0}}, X_{A_{0}}\right) \hookrightarrow\left(\operatorname{GSp}\left(W_{2}, \psi_{2}\right), S_{2}\right)$ of Shimura pairs, cf. 3.3 (ii). Let the 9-tuple $\left(E_{A_{0}}, g_{0}, \mathcal{A}_{g_{0}, 1, N_{0}}, \mathcal{N}_{0 N_{0}}, \overline{\mathcal{N}}_{0 N_{0}}, \mathcal{B}_{0}, \lambda_{\mathcal{B}_{0}}, \overline{\mathcal{B}}_{0}, K_{A_{0}}\left(N_{0}\right)\right)$ be the analogue of the 9 -tuple $\left(E_{A}, g, \mathcal{A}_{g, 1, N}, \mathcal{N}_{N}, \overline{\mathcal{N}}_{N}, \mathcal{B}, \lambda_{\mathcal{B}}, \overline{\mathcal{B}}, K_{A}(N)\right)$ formed by entries introduced in 2.4 and 2.5 , but obtained in the context of the triple $\left(f_{A_{0}}, L_{A_{0}}, N_{0}\right)$ instead of the triple $\left(f_{A}, L_{A}, N\right)$, for some number $N_{0} \in \mathbb{N} \backslash\{1,2\}$ that is relatively prime to p. Thus $E_{A_{0}}=E\left(H_{A_{0}}, X_{A_{0}}\right), 2 g_{0}=\operatorname{dim}_{\mathbb{Q}}\left(W_{2}\right)$, etc. From 2.4 .2 applied in the context of the new 7-tuple $\left(L_{A_{0}}, \mathcal{A}_{g_{0}, 1, N_{0}}, \mathcal{N}_{0 N_{0}}, \overline{\mathcal{N}}_{0 N_{0}}, \mathcal{B}_{0}, \lambda_{\mathcal{B}_{0}}, K_{A_{0}}\left(N_{0}\right)\right)$ instead of the 7 tuple $\left(L_{A}, \mathcal{A}_{g, 1, N}, \mathcal{N}_{N}, \overline{\mathcal{N}}_{N}, \mathcal{B}, \lambda_{\mathcal{B}}, K_{A}(N)\right)$, we get that we can view naturally $K_{1}$ as a $\mathbb{Q}$-algebra of $\mathbb{Q}$-endomorphisms of any pull pull back of $\mathcal{B}_{0}$ via some $\mathbb{C}$-valued point of $\operatorname{Sh}\left(H_{A_{0}}, X_{A_{0}}\right) / K_{A_{0}}\left(N_{0}\right)$. This implies that, up to a replacement of $N_{0}$ by a positive integral power of it, we can view $K_{1}$ as a $\mathbb{Q}$-algebra of $\mathbb{Q}$-endomorphisms of the pull back of $\mathcal{B}_{0}$ to the spectrum of the ring of fractions of $\mathcal{N}_{0 N_{0}}$ and thus also (cf. [10], Chapter I, Proposition 2.7) as a $\mathbb{Q}$-algebra of $\mathbb{Q}$-endomorphisms of either $\mathcal{B}_{0}$ or $\overline{\mathcal{B}}_{0}$.

The main point of the proof of 3.1 is to show that $\overline{\mathcal{N}}_{0 N_{0}} \backslash \mathcal{N}_{0 N_{0}}$ has no points of characteristic $p$ (the argument extends until 3.4.5). Let $k$ be an algebraic closure of the field $\mathbb{F}_{p}$ with $p$ elements. We have the following elementary criterion on when $\overline{\mathcal{N}}_{0 N_{0}} \backslash \mathcal{N}_{0 N_{0}}$ has no points of characteristic $p$.

\subsubsection{Lemma. We consider the following statement:}


$\mathfrak{Q}$ there exists a morphism $q: \operatorname{Spec}(k[[x]]) \rightarrow \overline{\mathcal{N}}_{0 N_{0}}$ with the property that it gives birth to morphisms $q_{\mathrm{sp}}: \operatorname{Spec}(k) \rightarrow \overline{\mathcal{N}}_{0 N_{0}}$ and $q_{\mathrm{gen}}: \operatorname{Spec}(k((x))) \rightarrow \overline{\mathcal{N}}_{0 N_{0}}$ that factor through $\overline{\mathcal{N}}_{0 N_{0}} \backslash \mathcal{N}_{0 N_{0}}$ and $\mathcal{N}_{0 N_{0}}$ (respectively).

If the statement $\mathfrak{Q}$ is false, then $\overline{\mathcal{N}}_{0 N_{0}} \backslash \mathcal{N}_{0 N_{0}}$ has no points of characteristic $p$.

Proof: We factor the projective morphism $\overline{\mathcal{N}}_{0 N_{0}} \rightarrow \operatorname{Spec}\left(O_{E_{A_{0}}}\left[\frac{1}{N_{0}}\right]\right)$ as the composite of a projective morphism $n_{A_{0}, N_{0}}: \overline{\mathcal{N}}_{0 N_{0}} \rightarrow \operatorname{Spec}\left(O_{E_{A_{0}, N_{0}}}\left[\frac{1}{N_{0}}\right]\right)$ with connected fibres and of a

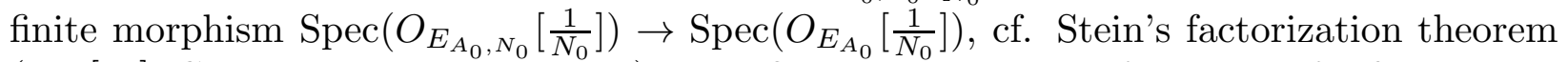
(see [14], Chapter III, Theorem 11.5); here $O_{E_{A_{0}, N_{0}}}$ is the ring of integers of a finite, étale $E_{A_{0}}$-algebra $E_{A_{0}, N_{0}}$. Let $\eta$ be an arbitrary point of $\operatorname{Spec}\left(O_{E_{A_{0}, N_{0}}}\left[\frac{1}{N_{0}}\right]\right)$ of characteristic $p$. Let $\mathcal{F}$ be the fibre of the morphism $n_{A_{0}, N_{0}}$ over $\eta$.

Let $E_{\eta}$ be the field that is a direct factor of $E_{A_{0}, N_{0}}$ and such that $\eta$ is a point of the spectrum of the direct factor $O_{E_{\eta}}\left[\frac{1}{N_{0}}\right]$ of $O_{E_{A_{0}, N_{0}}}\left[\frac{1}{N_{0}}\right]$. Let $\mathcal{E}$ be the fibre of $n_{A_{0}, N_{0}}$ over $\operatorname{Spec}\left(E_{\eta}\right)$; it is a connected component of $\mathcal{N}_{0 N_{0} E_{A_{0}}}=\overline{\mathcal{N}}_{0 N_{0} E_{A_{0}}}$ (cf. 2.7 (a) for the identity part). From the Zariski density part of 2.4 .3 applied in the context of $f_{A_{0}}$ instead of $f_{A}$, we get the existence of a finite field extension $E_{1 \eta}$ of $E_{\eta}$ such that we have a morphism $u: \operatorname{Spec}\left(E_{1 \eta}\right) \rightarrow \mathcal{N}_{0 N_{0} E_{A_{0}}}$ that factors through $\mathcal{E}$ and such that the Mumford-Tate group of a suitable (in fact of each) pull back of $u^{*}\left(\mathcal{B}_{0 E_{A_{0}}}\right)$ to $\mathbb{C}$, is a torus. As $\overline{\mathcal{N}}_{0 N_{0}}$ is a projective $O_{E_{A_{0}}}\left[\frac{1}{N_{0}}\right]$-scheme, the morphism $u$ extends to a morphism $\bar{u}: \operatorname{Spec}\left(O_{E_{1 \eta}}\left[\frac{1}{N_{0}}\right]\right) \rightarrow \overline{\mathcal{N}}_{0 N_{0}}$. We can assume that the field $E_{1 \eta}$ is such that the abelian variety $u^{*}\left(\mathcal{B}_{0 E_{A_{0}}}\right.$ ) extends (cf. 2.3.1) to an abelian scheme over $O_{E_{1 \eta}}\left[\frac{1}{N_{0}}\right]$ which (cf. [10], Chapter I, Proposition 2.7) is the semiabelian scheme $\bar{u}^{*}\left(\overline{\mathcal{B}}_{0}\right)$. From 2.5 (i) applied in the context of $\mathcal{A}_{g_{0}, 1, N_{0}}$, we get that $\bar{u}$ factors through $\mathcal{N}_{0 N_{0}}$. As $u$ factors through $\mathcal{E}, \operatorname{Im}(\bar{u})$ has a non-trivial intersection with $\mathcal{F}$. From the last two sentences, we get that the intersection $\mathcal{F} \cap \mathcal{N}_{0 N_{0}}$ is non-trivial.

But $\mathcal{F} \cap \mathcal{N}_{0 N_{0}}$ is an open subscheme of $\mathcal{F}$, cf. 2.5 (ii). As the statement $\mathfrak{Q}$ is false, the morphism $\mathcal{F} \cap \mathcal{N}_{0 N_{0}} \rightarrow \mathcal{F}$ is a closed embedding. So as $\mathcal{F}$ is connected and has a non-trivial intersection with $\mathcal{N}_{0 N_{0}}$, from the last two sentences we get that $\mathcal{F}=\mathcal{F} \cap \mathcal{N}_{0 N_{0}}$. Therefore $\mathcal{F}$ is a closed subscheme of $\mathcal{N}_{0 N_{0}}$. Thus $\overline{\mathcal{N}}_{0 N_{0}} \backslash \mathcal{N}_{0 N_{0}}$ has no points of characteristic $p$.

3.4.2. On $\mathfrak{Q}$. We will show that the assumption that there exists a morphism $q: \operatorname{Spec}(k[[x]]) \rightarrow \overline{\mathcal{N}}_{0 N_{0}}$ with the property that it gives birth to morphisms $q_{\mathrm{sp}}: \operatorname{Spec}(k) \rightarrow$ $\overline{\mathcal{N}}_{0 N_{0}}$ and $q_{\text {gen }}: \operatorname{Spec}(k((x))) \rightarrow \overline{\mathcal{N}}_{0 N_{0}}$ that factor through $\overline{\mathcal{N}}_{0 N_{0}} \backslash \mathcal{N}_{0 N_{0}}$ and $\mathcal{N}_{0 N_{0}}$ (respectively), leads to a contradiction (the argument will extend until 3.4.5). Let $C:=q^{*}\left(\overline{\mathcal{B}}_{0}\right)$; it is a semiabelian scheme over $k[[x]]$, whose generic fibre $C_{k((x))}$ is an abelian variety over $k((x))$ and whose special fibre $C_{k}$ is a semiabelian variety over $k$ that is not an abelian variety. The next two Subsubsections represent the moduli analogue of 3.1 (ii).

3.4.3. Proposition. The p-rank of $C_{k((x))}$ is at most $d_{0}\left(\right.$ see $3.3(v)$ for $\left.d_{0} \in \mathbb{N}\right)$.

Proof: Let $k_{1}:=\overline{k((x))}$. Let $B\left(k_{1}\right)$ be the field of fractions of the Witt ring $W\left(k_{1}\right)$ of $k_{1}$. Let $(M, \phi)$ be the $F$-isocrystal of $C_{k_{1}}$. Thus $M$ is a $B\left(k_{1}\right)$-vector space of dimension $2 g_{0}=\operatorname{dim}_{\mathbb{Q}}\left(W_{2}\right)$ that is equipped with a Frobenius-linear automorphism $\phi$. Let $O$ be a finite discrete valuation ring extension of $W\left(k_{1}\right)$ such that we have a morphism $q_{O}$ : $\operatorname{Spec}(O) \rightarrow \mathcal{N}_{0 N_{0}}$ with the property that it gives birth to a morphism $q_{k_{1}}: \operatorname{Spec}\left(k_{1}\right) \rightarrow$ $\mathcal{N}_{0 N_{0}}$ which factors through $q_{\text {gen }}$. By enlarging $O$, we can assume that the field of fractions 
$L$ of $O$ is naturally an algebra over the Galois extension of $\mathbb{Q}$ generated by $K_{1}$ and $E$; thus $K_{1} \otimes_{\mathbb{Q}} L \stackrel{\sim}{\rightarrow} L^{\left[K_{1}: \mathbb{Q}\right]}$ and therefore the set $\operatorname{Hom}\left(K_{1}, L\right)$ has $\left[K_{1}: \mathbb{Q}\right]$ elements.

Let $D:=q_{O}^{*}(\mathcal{B})$; it is an abelian scheme over $O$. We fix an embedding $i_{L}: L \hookrightarrow \mathbb{C}$ that extends $i_{E}$; thus we can speak about $D_{\mathbb{C}}$. Viewing $K_{1}$ as a $\mathbb{Q}$-algebra of $\mathbb{Q}$-endomorphisms of the pull back $D_{L}$ of $\mathcal{B}_{0}$ (cf. 3.4), we get that we have a natural $\mathbb{Q}_{p}$-monomorphism $K_{1} \otimes_{\mathbb{Q}} \mathbb{Q}_{p} \hookrightarrow \operatorname{End}(M, \phi)$. Thus $M$ has a natural structure of a $K_{1} \otimes_{\mathbb{Q}} B\left(k_{1}\right)$-module and so also of an $F_{1} \otimes_{\mathbb{Q}} \mathbb{Q}_{p}$-module. As $F_{1} \otimes_{\mathbb{Q}} \mathbb{Q}_{p}=\prod_{j \in J} F_{1 j}$, we have a unique decomposition

$$
(M, \phi)=\oplus_{j \in J}\left(M_{j}, \phi\right)
$$

with the property that each $M_{j}$ is an $F_{1 j}$-vector space.

We will apply 2.4 .2 in the context of $\left(L_{A_{0}}, \mathcal{A}_{g_{0}, 1, N_{0}}, \mathcal{N}_{0 N_{0}}, \overline{\mathcal{N}}_{0 N_{0}}, \mathcal{B}_{0}, \lambda_{\mathcal{B}_{0}}, K_{A_{0}}\left(N_{0}\right)\right)$ instead of $\left(L_{A}, \mathcal{A}_{g, 1, N}, \mathcal{N}_{N}, \overline{\mathcal{N}}_{N}, \mathcal{B}, \lambda_{\mathcal{B}}, K_{A}(N)\right)$. Thus we can identify $H_{1}(D(\mathbb{C}), \mathbb{Q})=W_{2}$ and $H_{e t}^{1}\left(D_{\bar{L}}, \mathbb{Q}_{p}\right)=W_{2}^{*} \otimes_{\mathbb{Q}} \mathbb{Q}_{p}$ and these identifications are compatible with the natural $K_{1^{-}}$ actions. Let $B_{\text {crys }}$ be the crystalline Fontaine ring of $L$; it is an integral $B\left(k_{1}\right)$-algebra (see $[11])$. From the functorial aspect of Fontaine comparison theory we get that the two $F_{1} \otimes_{\mathbb{Q}}$ $B_{\text {crys }}$-modules $W_{2}^{*} \otimes_{\mathbb{Q}} B_{\text {crys }}=H_{e t}^{1}\left(D_{\bar{L}}, \mathbb{Q}_{p}\right) \otimes_{\mathbb{Q}_{p}} B_{\text {crys }}$ and $M \otimes_{B\left(k_{1}\right)} B_{\text {crys }}$ are isomorphic (see [12], Theorem of 6.1.4). This implies that we have $\operatorname{dim}_{B\left(k_{1}\right)}\left(M_{j}\right)=\operatorname{dim}_{\mathbb{Q}_{p}}\left(W_{2 j}\right)$ for all $j \in J$, cf. $(2)$ and $3.3(\mathrm{v})$. From this and the inequality of $3.3(\mathrm{v})$, we get that $\sum_{j \in J \backslash\left\{j_{0}\right\}} \operatorname{dim}_{B\left(k_{1}\right)}\left(M_{j}\right) \leq d_{0}$. The $p$-rank of $C_{k((x))}$ is the multiplicity of the slope 0 for the $F$-isocrystal $(M, \phi)$. Thus, if the $F$-isocrystal $\left(M_{j_{0}}, \phi\right)$ does not have slope 0 with positive multiplicity, then the $p$-rank of $C_{k((x))}$ is at most $\sum_{j \in J \backslash\left\{j_{0}\right\}} \operatorname{dim}_{B\left(k_{1}\right)}\left(M_{j}\right) \leq d_{0}$.

We show that the assumption that $\left(M_{j_{0}}, \phi\right)$ has slope 0 with positive multiplicity leads to a contradiction. Let $M_{j_{0}}^{00}$ be the $\mathbb{Q}_{p}$-vector subspace of $M_{j_{0}}$ formed by elements fixed by $\phi$. Thus $M_{j_{0}}^{0}:=M_{j_{0}}^{00} \otimes_{\mathbb{Q}_{p}} B\left(k_{1}\right)$ is the maximal $B\left(k_{1}\right)$-vector subspace of $M_{j_{0}}$ that is normalized by $\phi$ and such that all slopes of $\left(M_{j_{0}}^{0}, \phi\right)$ are 0 . We have $\operatorname{dim}_{B\left(k_{1}\right)}\left(M_{j_{0}}^{0}\right) \geq 1$. Obviously $M_{j_{0}}^{00}$ is a $K_{1} \otimes_{\mathbb{Q}} \mathbb{Q}_{p}$-module and thus $K_{1 j_{0}}$ acts on $M_{j_{0}}^{00}$. As $M_{j_{0}}$ is an $F_{1 j_{0}}$-vector space and as $K_{1 j_{0}}$ is a field (see 3.2.3), $M_{j_{0}}^{00}$ is a $K_{1 j_{0}}$-vector space.

Let $F_{L}^{1}$ be the direct summand of $M \otimes_{B\left(k_{1}\right)} L$ that defines the Hodge filtration of $M \otimes_{B\left(k_{1}\right)} L$ associated to $D_{L}$ via the functorial identification $M \otimes_{B\left(k_{1}\right)} L=H_{d R}^{1}\left(D_{L} / L\right.$ ) (see [5], Theorem 1.3). As $D$ is an abelian scheme over $O$, the triple $\left(M, \phi, F_{L}^{1}\right)$ is an admissible filtered module over $L$ in the sense of [12], Subsubsection 5.5.2 (cf. [12], Theorem of 6.1.4) and thus it is also a weakly-admissible filtered module over $L$ in the sense of [12], Definition 4.4 .3 (cf. [12], Subsubsection 5.5.3). This implies that the Hodge polygon $\mathfrak{p}_{H}$ of $\left(M_{j_{0}}^{0}, F_{L}^{1} \cap M_{j_{0}}^{0}\right)$ is below the Newton polygon $\mathfrak{p}_{N}$ of $\left(M_{j_{0}}^{0}, \phi\right)$, cf. [12], Proposition 4.4.2. As $\mathfrak{p}_{N}$ has all slopes 0 , we get that in fact $\mathfrak{p}_{H}=\mathfrak{p}_{N}$. Thus $M_{j_{0}}^{0} \otimes_{B\left(k_{1}\right)} L \cap F_{L}^{1}=0$.

In what follows we fix an algebraic closure $\bar{L}$ of $L$ and we identify (to be compared with 3.2.2) $\overline{\mathbb{Q}}$ and $\overline{\mathbb{Q}_{p}}$ with their algebraic closures in $\bar{L}$. Thus we also identify the set $\operatorname{Hom}\left(F_{1}, \overline{\mathbb{Q}}\right)=\operatorname{Hom}\left(F_{1}, \mathbb{R}\right)$ with the two sets $\operatorname{Hom}\left(F_{1}, \overline{\mathbb{Q}_{p}}\right)$ and $\operatorname{Hom}\left(F_{1}, L\right)=\operatorname{Hom}\left(F_{1}, \bar{L}\right)$ (resp. we identify the sets $\operatorname{Hom}\left(K_{1}, \overline{\mathbb{Q}}\right)=\operatorname{Hom}\left(K_{1}, \mathbb{C}\right)$ and $\operatorname{Hom}\left(K_{1}, L\right)=\operatorname{Hom}\left(K_{1}, \bar{L}\right)$ ).

Let $I_{1, p}^{(2)}\left(\right.$ resp. $\left.I_{1, p}\right)$ be the non-empty subset of $\operatorname{Hom}\left(K_{1}, L\right)\left(\operatorname{resp}\right.$. of $\left.\operatorname{Hom}\left(F_{1}, L\right)\right)$ formed by embeddings $K_{1} \hookrightarrow L$ (resp. $F_{1} \hookrightarrow L$ ) that have the property that the local ring $O$ of $L$ dominates the ring of integers of $F_{1 j_{0}}$ in such a way that the resulting embedding $F_{0 j_{0}} \hookrightarrow \bar{L}$ is defined by the embedding $F_{0 j_{0}} \hookrightarrow \overline{\mathbb{Q}_{p}}$ that defines (cf. 3.2.2) the element $i_{0} \in$ 
$\operatorname{Hom}\left(F_{0}, \mathbb{R}\right)$. As $K_{1 j_{0}}$ is a quadratic field extension of $F_{1 j_{0}}$, the subset $I_{1, p}^{(2)}$ of $\operatorname{Hom}\left(K_{1}, L\right)$ is $\operatorname{Gal}\left(K_{1} / F_{1}\right)$-invariant and $I_{1, p}$ is naturally identified with the quotient of $I_{1, p}^{(2)}$ under the action of $\operatorname{Gal}\left(K_{1} / F_{1}\right)$ on it. Let

$$
M_{j_{0}}^{00} \otimes_{\mathbb{Q}_{p}} L=M_{j_{0}}^{0} \otimes_{B\left(k_{1}\right)} L=\oplus_{i_{1, L} \in \operatorname{Hom}\left(K_{1}, L\right)} M_{j_{0}}^{0 i_{1, L}}
$$

be the natural decomposition into $K_{1} \otimes_{\mathbb{Q}} L$-modules. As $M_{j_{0}}^{00}$ is a $K_{1 j_{0}}$-vector space, each $M_{j_{0}}^{0 i_{1, L}}$ with $i_{1, L} \in I_{1, p}^{(2)}$ is a non-trivial $L$-vector space.

From (1) and the identification $M \otimes_{B\left(k_{1}\right)} L=H_{d R}^{1}\left(D_{L} / L\right)$, we get that we have natural identifications of (cf. 3.3 (iii)) $K_{1}$-vector spaces

$M \otimes_{B\left(k_{1}\right)} \mathbb{C}=H_{d R}^{1}\left(D_{\mathbb{C}} / \mathbb{C}\right)=H^{1}(D(\mathbb{C}), \mathbb{Q}) \otimes_{\mathbb{Q}} \mathbb{C}=W_{2}^{*} \otimes_{\mathbb{Q}} \mathbb{C}=\oplus_{i \in \operatorname{Hom}\left(F_{1}, \mathbb{R}\right)} W\left(i_{1}\right)^{*} \oplus W\left(i_{2}\right)^{*}$,

under which $F_{L}^{1} \otimes_{L} \mathbb{C}$ gets identified with the Hodge filtration of $W_{2}^{*} \otimes_{\mathbb{Q}} \mathbb{C}$ defined by a point $x_{2} \in X_{0} \subseteq X_{2}$ that is naturally associated to $D_{\mathbb{C}}$.

Under the identification $\operatorname{Hom}\left(F_{1}, \mathbb{R}\right)=\operatorname{Hom}\left(F_{1}, L\right)$, to the subset $I_{1, p}$ of $\operatorname{Hom}\left(F_{1}, L\right)$ corresponds a non-empty subset $I_{1}$ of $\operatorname{Hom}\left(F_{1}, \mathbb{R}\right)$ formed by elements that extend the embedding $i_{0}: F_{0} \hookrightarrow \mathbb{R}$ of 3.2. Let $i \in I_{1}$. Let $i_{1}, i_{2}: K_{1} \hookrightarrow \mathbb{C}$ be the two embeddings that extend $i$ and that are listed in the same order as in the proof of 3.3. As the set $I_{1, p}^{(2)}$ is $\operatorname{Gal}\left(K_{1} / F_{1}\right)$-invariant, there exist elements $i_{1, p}$ and $i_{2, p}$ of $I_{1, p}^{(2)}$ that correspond to $i_{1}$ and $i_{2}$ (respectively) via the identification $\operatorname{Hom}\left(K_{1}, \mathbb{C}\right)=\operatorname{Hom}\left(K_{1}, L\right)$. From 3.3 (viii) applied to $i \in I_{1} \subseteq \operatorname{Hom}\left(F_{1}, \mathbb{R}\right)$, we get that the identifications of (3) give birth to inclusions

$$
M_{j_{0}}^{0 i_{1, p}} \otimes_{B\left(k_{1}\right)} \mathbb{C} \subseteq W\left(i_{1}\right)^{*} \subseteq F_{L}^{1} \otimes_{L} \mathbb{C}
$$

Thus $M_{j_{0}}^{0 i_{1, p}} \otimes_{B\left(k_{1}\right)} L \subseteq F_{L}^{1}$. Therefore $M_{j_{0}}^{0} \otimes_{B\left(k_{1}\right)} L \cap F_{L}^{1} \supseteq M_{j_{0}}^{0 i_{1, p}} \otimes_{B\left(k_{1}\right)} L \supsetneqq 0$. This contradicts the identity $M_{j_{0}}^{0} \otimes_{B\left(k_{1}\right)} L \cap F_{L}^{1}=0$. Thus the $F$-isocrystal $\left(M_{j_{0}}, \phi\right)$ does not have slope 0 with positive multiplicity. Therefore the $p$-rank of $C_{k((x))}$ is at most $d_{0}$. $\square$

3.4.4. The study of $C$. We now use 3.4.3 to reach the contradiction promised in 3.4.2. Let $T_{k}$ be the maximal torus of the semiabelian variety $C_{k}$. As $C_{k}$ is not an abelian scheme, we have $1 \leq \operatorname{dim}\left(T_{k}\right)$. Let $K_{1 \mathbb{Z}}:=K_{1} \cap \operatorname{End}(C)$ (the intersection being taken inside $\left.\operatorname{End}(C) \otimes_{\mathbb{Z}} \mathbb{Q}\right)$; it is a $\mathbb{Z}$-order of $K_{1}$. As $K_{1 \mathbb{Z}}$ acts on $C$, it also acts on $T_{k}$ and thus also on the free $\mathbb{Z}$-module $X_{*}\left(T_{k}\right)$ of cocharacters of $T_{k}$. Let $m, l \in \mathbb{N}$.

There exists a unique torus $T_{k, l}$ of $C_{k[[x]] /\left(x^{l}\right)}$ which lifts $T_{k}$, cf. [9], Exp. IX, Theorem 3.6 bis. Loc. cit. implies that we have a canonical identification $T_{k, l}=T_{k} \times_{k} k[[x]] /\left(x^{l}\right)$ that lifts the identity automorphism of $T_{k}$. Thus $T_{k, l}\left[p^{m}\right]=T_{k}\left[p^{m}\right]_{k[[x]] /\left(x^{l}\right)}$ is naturally a closed subgroup scheme of $C_{k[[x]] /\left(x^{l}\right)}\left[p^{m}\right]$. Due the uniqueness property of $T_{k, l}$, the torus $T_{k, l+1}$ lifts $T_{k, l}$. Thus by passing to the limit $l \rightarrow \infty$, we get that $T_{k}\left[p^{m}\right]_{k[[x]]}$ is naturally identified with a closed subgroup scheme of $C_{k[[x]]}\left[p^{m}\right]$ and thus that $T_{k}\left[p^{m}\right]_{k((x))}$ is naturally identified with a closed subgroup scheme of $C_{k((x))}\left[p^{m}\right]$. To check that these last identifications are functorial, it suffices to show that for each closed, semiabelian subscheme $C^{\prime}$ of $C^{2}$, the unique subtorus $T_{k, l}^{\prime}$ of $C_{k[[x]] /\left(x^{l}\right)}^{\prime}$ that lifts the maximal torus $T_{k}^{\prime}$ 
of $C_{k}^{\prime}$, is a subtorus of $T_{k, l}^{2}$. As $T_{k}^{\prime}$ is a subtorus of $T_{k}^{2}$, from the uniqueness part of loc. cit. we get that: (i) there exists a unique subtorus $T_{k, l}^{\prime \prime}$ of $T_{k, l}^{2}$ that lifts $T_{k}^{\prime}$, and (ii) we have an identity $T_{k, l}^{\prime}=T_{k, l}^{\prime \prime}$ of subtori of $C_{k[[x]] /\left(x^{l}\right)}^{2}$. Thus $T_{k, l}^{\prime}$ is a subtorus of $T_{k, l}^{2}$.

The closed embedding homomorphism $\theta_{m}: T_{k}\left[p^{m}\right]_{k((x))} \hookrightarrow C_{k((x))}\left[p^{m}\right]$ is compatible with the natural $K_{1 \mathbb{Z}}$-actions, cf. previous paragraph. The existence of $\theta_{m}$ implies that the $p$-rank of $C_{k((x))}$ is at lest $\operatorname{dim}\left(T_{k}\right)$. Thus we have $\operatorname{dim}\left(T_{k}\right) \leq d_{0}$, cf. 3.4.3. We get a sequence of inequalities and equalities

$$
\operatorname{rk}_{\mathbb{Z}}\left(X_{*}\left(T_{k}\right)\right)=\operatorname{dim}\left(T_{k}\right) \leq d_{0}<2\left[F_{1}: \mathbb{Q}\right]=\left[K_{1}: \mathbb{Q}\right]=\operatorname{rk}_{\mathbb{Z}}\left(K_{1 \mathbb{Z}}\right)
$$

As $\operatorname{rk}_{\mathbb{Z}}\left(X_{*}\left(T_{k}\right)\right)<\operatorname{rk}_{\mathbb{Z}}\left(K_{1 \mathbb{Z}}\right)$, the action of $K_{1 \mathbb{Z}}$ on $X_{*}\left(T_{k}\right)$ is trivial. Thus $K_{1 \mathbb{Z}}$ acts trivially on $T_{k}$ and so also on $\operatorname{Im}\left(\theta_{m}\right)$. As $\theta_{m}$ is compatible with the $K_{1 \mathbb{Z}}$-actions, by taking $m \rightarrow \infty$ we get that $K_{1 \mathbb{Z}}$ acts trivially on a quotient of $M$ of dimension $\operatorname{dim}\left(T_{k}\right) \geq 1$. This contradicts the fact that (cf. (3)) $M$ is a $K_{1}$-vector space. Thus the assumption of 3.4.2 that the statement $\mathfrak{Q}$ is true, leads to a contradiction. So the statement $\mathfrak{Q}$ is false.

3.4.5. End of the proof of 3.1. As the statement $\mathfrak{Q}$ is false, the complement $\overline{\mathcal{N}}_{0 N_{0}} \backslash \mathcal{N}_{0 N_{0}}$ has no points of characteristic $p$ (cf. 3.4.1). We recall that $\overline{\mathcal{N}}_{0 N_{0}}$ is a projective $O_{E_{A_{0}}}\left[\frac{1}{N_{0}}\right]$ scheme and that (cf. 2.7 (a) applied in the context of $f_{A_{0}}$ ) we have $\overline{\mathcal{N}}_{0 N_{0} E_{A_{0}}}=\mathcal{N}_{0 N_{0} E_{A_{0}}}$. From the last two sentences, we get that, by replacing $N_{0}$ with a multiple of it by a natural number prime to $p$, we can assume that in fact we have $\mathcal{N}_{0 N_{0}}=\overline{\mathcal{N}}_{0 N_{0}}$.

By replacing $E$ with a finite field extension of it, we can assume (see proof of 2.6) that there exists a morphism $u_{A}: \operatorname{Spec}(E) \rightarrow \mathcal{N}_{N}$ such that $\left(A, \lambda_{A}\right)=u_{A}^{*}\left(\mathcal{B}, \lambda_{\mathcal{B}}\right)$ and:

(i) the composite of the morphism $\operatorname{Spec}(\mathbb{C}) \rightarrow \operatorname{Spec}(E)$ defined by $i_{E}$ with $u_{A}$ is the point of $\mathcal{N}_{N}(\mathbb{C})=\operatorname{Sh}\left(H_{A}, X_{A}\right) / K_{A}(N)(\mathbb{C})=H_{A}(\mathbb{Q}) \backslash X_{A} \times H_{A}\left(\mathbb{A}_{f}\right) / K_{A}(N)$ defined by the equivalence class $\left[h_{A}, 1_{W_{A}}\right]$ (here $1_{W_{A}}$ is the identity element of $H_{A}\left(\mathbb{A}_{f}\right)$ ).

By replacing $N$ and $N_{0}$ with multiplies of them by natural numbers prime to $p$, we can assume that there exists a compact, open subgroup $K_{0}$ of $H_{0}\left(\mathbb{A}_{f}\right)$ such that the images of both $K_{A}(N)$ and $K_{A_{0}}\left(N_{0}\right)$ in $H_{0}\left(\mathbb{A}_{f}\right)$, are contained in $K_{0}$. We have functorial morphisms $\operatorname{Sh}\left(H_{A}, X_{A}\right) / K_{A}(N) \rightarrow \operatorname{Sh}\left(H_{0}, X_{0}\right) / K_{0}$ and $\operatorname{Sh}\left(H_{A_{0}}, X_{A_{0}}\right) / K_{A_{0}}\left(N_{0}\right) \rightarrow \operatorname{Sh}\left(H_{0}, X_{0}\right) / K_{0}$, the last one being finite. Based on this and (i), by replacing $E$ with a finite field extension of it, we can assume that there exists a morphism $u_{A_{0}}: \operatorname{Spec}(E) \rightarrow \mathcal{N}_{0 N_{0}}$ such that the $E$-valued points of $\operatorname{Sh}\left(H_{0}, X_{0}\right) / K_{0}$ defined naturally by $u_{A}$ and $u_{A_{0}}$ coincide and moreover:

(ii) the composite of the morphism $\operatorname{Spec}(\mathbb{C}) \rightarrow \operatorname{Spec}(E)$ defined by $i_{E}$ with $u_{A_{0}}$ is the point of $\mathcal{N}_{0 N_{0}}(\mathbb{C})=\operatorname{Sh}\left(H_{A_{0}}, X_{A_{0}}\right) / K_{A_{0}}\left(N_{0}\right)(\mathbb{C})=H_{A_{0}}(\mathbb{Q}) \backslash X_{A_{0}} \times H_{A_{0}}\left(\mathbb{A}_{f}\right) / K_{A_{0}}\left(N_{0}\right)$ defined by the equivalence class $\left[h_{A_{0}}, 1_{W_{2}}\right]$ (here $1_{W_{2}}$ is the identity element of $H_{A_{0}}\left(\mathbb{A}_{f}\right)$ ).

Let $\left(A_{0}, \lambda_{A_{0}}\right):=u_{A_{0}}^{*}\left(\mathcal{B}_{0}, \lambda_{\mathcal{B}_{0}}\right)$. We can identify naturally the triple $\left(W_{2}, \psi_{2}, X_{2}\right)$ with $\left(W_{A_{0}}, \psi_{A_{0}}, X_{A_{0}}\right)$ and thus the notations for $\left(A_{0}, \lambda_{A_{0}}\right)$ and for the following 9-tuple $\left(E_{A_{0}}, g_{0}, \mathcal{A}_{g_{0}, 1, N_{0}}, \mathcal{N}_{0 N_{0}}, \overline{\mathcal{N}}_{0 N_{0}}, \mathcal{B}_{0}, \lambda_{\mathcal{B}_{0}}, \overline{\mathcal{B}}_{0}, K_{A_{0}}\left(N_{0}\right)\right)$ match. Based on (ii) and the definition of $H_{A_{0}}$ in the beginning of 3.4, we get that the Mumford-Tate group of $A_{0 \mathbb{C}}$ is $H_{A_{0}}$. Thus, as $h_{A_{0}}$ lifts $h_{A 0}, 3.1$ (a) holds. As $\mathcal{N}_{0 N_{0}}=\overline{\mathcal{N}}_{0 N_{0}}$, condition 3.1 (b) also holds. This ends the proof of 3.1. As $\mathcal{N}_{0 N_{0}}=\overline{\mathcal{N}}_{0 N_{0}}$, it is easy to see that 3.1 (ii) holds. As we have a natural monomorphism $K_{1} \hookrightarrow \operatorname{End}\left(A_{0 \mathbb{C}}\right)($ cf. 3.4$)$ and as $\left[K_{1}: \mathbb{Q}\right]>2 d_{0}, 3.1$ (i) also holds. 


\section{$\S 4$. Proof of the Basic Theorem 1.2, examples, and applications}

In 4.1 we proof 1.2. In 4.2 we include two examples. In 4.3 we obtain an equivalent form of 1.2. In 4.4 to 4.6 we apply 4.3 to Néron models and to integral models of Shimura varieties of preabelian type. We use the notations of the first paragraph of $\S 1$ and of 2.4.

4.1. Proof of 1.2. To prove 1.2 , we can assume that $A$ has a principal polarization $\lambda_{A}$ (cf. 2.3) and that $H_{A}^{\text {ad }}$ is a non-trivial group (cf. 2.3.1). Let $N_{A} \in \mathbb{N}$ be such that $A$ extends to an abelian scheme over $O_{E}\left[\frac{1}{N_{A}}\right]$. Suppose that for each prime divisor $p$ of $N_{A}$, there exists a finite field extension $E_{p}$ of $E$ such that $A_{E_{p}}$ has good reduction with respect to all primes of $E_{p}$ that divide $p$. If $E_{1}$ is the composite field of $E$ and of all the fields $E_{p}$ 's with $p$ a prime divisor of $N_{A}$, then $A_{E_{1}}$ extends to an abelian scheme over $O_{E_{1}}$. Thus to end the proof of 1.2 , we only need to show that $E_{p}$ exists for all prime divisors $p$ of $N_{A}$.

To check this, we can replace $E$ by any finite field extension of it. Let

$$
\left(H_{A}^{\mathrm{ad}}, X_{A}^{\mathrm{ad}}\right)=\prod_{t \in \mathfrak{T}}\left(H_{t}, X_{t}\right)
$$

be the product decomposition into simple, adjoint Shimura pairs. By replacing $E$ with a finite field extension of it, based on 3.1 we can assume that for each $t \in \mathfrak{T}$ there exists a principally polarized abelian variety $\left(A_{t}, \lambda_{A_{t}}\right)$ over $E$ such that:

(i) we have an identity $\left(H_{A_{t}}^{\mathrm{ad}}, X_{A_{t}}^{\mathrm{ad}}\right)=\left(H_{t}, X_{t}\right)$ with the property that the homomorphism $h_{A_{t}}^{\text {ad }}: \mathbb{S} \rightarrow H_{A_{t} \mathbb{R}}^{\text {ad }}=H_{t \mathbb{R}}$ is the homomorphism $h_{A t}$ of 2.4.1, and

(ii) condition 2.6 (b) holds in the context of $\left(A_{t}, \lambda_{A_{t}}\right)$, for some number $N_{t} \in \mathbb{N} \backslash\{1,2\}$ that is relatively prime to $p$.

Let $E_{p, t}$ be a finite field extension of $E$ such that $A_{t E_{p, t}}$ has good reduction with respect to all primes of $E_{p, t}$ that divide $p$, cf. (ii) and the last part of 2.6 applied to $A_{t}$. Let $\tilde{E}_{p}$ be the composite field of $E_{p, t}$ 's, with $t \in \mathfrak{T}$. Let $B:=\prod_{t \in \mathfrak{T}} A_{t}$; it is an abelian variety over $E$ with the property that $B_{\tilde{E}_{p}}$ has good reduction with respect to all primes of $\tilde{E}_{p}$ that divide $p$. But $H_{A}^{\text {ad }}$ is the smallest subgroup of $H_{A}^{\text {ad }}=\prod_{t \in \mathfrak{T}} H_{t}$ with the property that $h_{A}^{\text {ad }}=\prod_{t \in \mathfrak{T}} h_{A t}$ factors through $H_{A \mathbb{R}}^{\text {ad }}$, cf. the definition of $H_{A}$. The Mumford-Tate group $H_{B}$ is a subgroup of $\prod_{t \in \mathfrak{T}} H_{A_{t}}$ that surjects into all groups $H_{A_{t}}$. This implies that $H_{B}^{\text {ad }}$ is the smallest subgroup of $\prod_{t \in \mathfrak{T}} H_{A_{t}}^{\text {ad }}$ with the property that $h_{B}^{\text {ad }}=\prod_{t \in \mathfrak{T}} h_{A_{t}}^{\text {ad }}$ factors through $H_{B \mathbb{R}}^{\text {ad }}$. From the last two sentences and (i) we get that:

(iii) we have identifications $\left(H_{B}^{\mathrm{ad}}, X_{B}^{\mathrm{ad}}\right)=\prod_{t \in \mathfrak{T}}\left(H_{t}, X_{t}\right)=\left(H_{A}^{\mathrm{ad}}, X_{A}^{\mathrm{ad}}\right)$ with the property that the homomorphism $h_{B}^{\text {ad }}: \mathbb{S} \rightarrow H_{B \mathbb{R}}^{\text {ad }}=H_{A \mathbb{R}}^{\text {ad }}$ is the homomorphism $h_{A}^{\text {ad }}$ of 2.4 .

The reductive group $H_{A \times_{E} B}$ is a subgroup of $H_{A} \times_{\mathbb{Q}} H_{B}$ whose adjoint is (cf. (iii)) isomorphic to $H_{A}^{\text {ad }}=H_{B}^{\text {ad }}$. As $B_{\tilde{E}_{p}}$ has good reduction with respect to all primes of $\tilde{E}_{p}$ that divide $p$, from (iii) and [27], Proposition 4.1.2 we get that there exists a finite field extension $E_{p}$ of $\tilde{E}_{p}$ such that $A_{E_{p}}$ has good reduction with respect to all primes of $E_{p}$ that divide $p$. So the field $E_{p}$ exists for each prime divisor $p$ of $N_{A}$. This ends the proof of 1.2. 
4.2. Two examples. We include two examples that make the connection between the results of this paper and of [27]. The first one points out simple, completely new situations to which the Basic Theorem applies. The second one pertains to [27], Proposition 4.2.13.

4.2.1. Example. Let $F$ be a totally real, cubic, Galois extension of $\mathbb{Q}$; for instance, we can take $F$ to be $\mathbb{Q}\left(\zeta_{7}+\zeta_{7}^{-1}\right)$, where $\zeta_{7}$ is a primitive root of 1 of order 7 . We assume that $H_{A}^{\text {ad }}$ is a simple group of the form $\operatorname{Res}_{F / \mathbb{Q}} G$ for some absolutely simple, adjoint group $G$ over $F$ of $B_{n}$ (resp. of $D_{n}$ ) Dynkin type with $n \geq 2$ (resp. with $n \geq 4$ ). We also assume that the product decomposition $H_{A \mathbb{R}}^{\text {ad }}=\mathcal{F}_{1} \times_{\mathbb{R}} \mathcal{F}_{2} \times_{\mathbb{R}} \mathcal{F}_{3}$ into simple factors is such that $\mathcal{F}_{1}$ and $\mathcal{F}_{2}$ are non-compact and $\mathcal{F}_{3}$ is compact. Thus $A$ has compact factors.

We identify $\operatorname{Lie}\left(H_{A \mathbb{C}}^{\text {ad }}\right)$ with $\operatorname{Lie}\left(H_{A \mathbb{C}}^{\text {der }}\right)$. We check that the representation of $\operatorname{Lie}\left(H_{A \mathbb{C}}^{\text {ad }}\right)$ on $W_{A} \otimes_{\mathbb{Q}} \mathbb{C}$ is a direct sum of irreducible representations of single simple factors of $\operatorname{Lie}\left(H_{A \mathbb{C}}^{\text {ad }}\right)$. We show that the assumption that this is not true, leads to a contradiction. This assumption implies that there exists $s \in\{2,3\}$ and there exist distinct simple factors $\mathcal{L}_{1}, \ldots, \mathcal{L}_{s}$ of $\operatorname{Lie}\left(H_{A \mathbb{C}}^{\mathrm{ad}}\right)$, such that a simple $\operatorname{Lie}\left(H_{A \mathbb{C}}^{\mathrm{ad}}\right)$-submodule $\mathcal{W}_{0}$ of $W_{A} \otimes_{\mathbb{Q}} \mathbb{C}$ is a tensor product $\mathcal{W}_{1} \otimes_{\mathbb{C}} \cdots \otimes_{\mathbb{C}} \mathcal{W}_{s}$, where $\mathcal{W}_{r}$ is a simple $\mathcal{L}_{r}$-module for all $r \in\{1, \ldots, s\}$. As the representation of $\operatorname{Lie}\left(H_{A \mathbb{C}}^{\text {ad }}\right)$ on $W_{A} \otimes_{\mathbb{Q}} \mathbb{C}$ is defined over $\mathbb{Q}$ and as $F$ is a cubic Galois extension of $\mathbb{Q}$, we can choose $\mathcal{W}_{0}$ such that we have $\mathcal{L}_{1}=\operatorname{Lie}\left(\mathcal{F}_{1 \mathbb{C}}\right)$ and $\mathcal{L}_{2}=\operatorname{Lie}\left(\mathcal{F}_{2 \mathbb{C}}\right)$. As the images of $\mu_{A}$ in $\mathcal{F}_{1 \mathbb{C}}$ and $\mathcal{F}_{2 \mathbb{C}}$ are non-trivial, for $s=2$ (resp. for $s=3$ ) the Hodge filtration $\left(F^{a}\left(\mathcal{W}_{0}\right)\right)_{a \in \mathbb{Z}}$ of $\mathcal{W}_{0}$ defined by $\mu_{A}$ is the tensor product of non-trivial Hodge filtrations of $\mathcal{W}_{1}$ and $\mathcal{W}_{2}$ (resp. is the tensor product of non-trivial Hodge filtrations of $\mathcal{W}_{1}$ and $\mathcal{W}_{2}$ and of a trivial Hodge filtration of $\mathcal{W}_{3}$ ); here by a trivial filtration of $W_{r}$ we mean a filtration of $W_{r}$ that does not contain any proper subspace of $W_{r}$. We easily get that there exists $a \in \mathbb{Z} \backslash\{-1,0\}$ such that $F^{a}\left(\mathcal{W}_{0}\right) / F^{a+1}\left(\mathcal{W}_{0}\right) \neq 0$. Thus $\mu_{A}$ does not act on $F^{a}\left(\mathcal{W}_{0}\right) / F^{a+1}\left(\mathcal{W}_{0}\right)$ via either the trivial or the identical character of $\mathbb{G}_{m \mathbb{C}}$. Contradiction.

As the representation of $\operatorname{Lie}\left(H_{A \mathbb{C}}^{\mathrm{ad}}\right)$ on $W_{A} \otimes_{\mathbb{Q}} \mathbb{C}$ is a direct sum of irreducible representations of single simple factors of $\operatorname{Lie}\left(H_{A \mathbb{C}}^{\mathrm{ad}}\right)$, from [27], Proposition 2.2.3 we get that the results of [27], Subsection 2.4 do not imply the existence of a finite field extension $E_{1}$ of $E$ such that $A_{E_{1}}$ extends to an abelian scheme over $O_{E_{1}}$.

4.2.2. Example. Suppose $A$ is such that there exists a prime $p \in \mathbb{N}$ with the property that $H_{A \mathbb{Q}_{p}}^{\text {ad }}$ is anisotropic (i.e., its $\mathbb{Q}_{p}$-rank is 0). This implies that the $\mathbb{Q}$-rank of $H_{A}^{\text {ad }}$ is also 0 . As $H_{A \mathbb{Q}_{p}}^{\text {ad }}$ is anisotropic, the group $H_{A \mathbb{Q}_{p}}^{\text {ad }}\left(\mathbb{Q}_{p}\right)$ is compact and does not have non-trivial unipotent elements (see [28], Subsection 3.4). So also the group $H_{A \mathbb{Q}_{p}}\left(\mathbb{Q}_{p}\right)$ does not have non-trivial unipotent elements. Thus from [27], Theorem 1.6.1 we get that there exists a finite field extension $E_{1}$ of $E$ such that $A_{E_{1}}$ extends to an abelian scheme over $O_{E_{1}}$.

From the classification of simple adjoint groups over $p$-adic fields (see [29], Table II, pp. 55-58) and the fact that any simple factor of $H_{A \mathbb{C}}^{\text {ad }}$ is of classical Lie types, one gets that the assumption that $H_{A \mathbb{Q}_{p}}^{\text {ad }}$ is anisotropic implies that each simple factor of $H_{A \mathbb{C}}^{\text {ad }}$ is of $A_{n}$ Lie type for some $n \in \mathbb{N}$. Thus the last sentence of the previous paragraph is also implied by the combination of [32], Corollary 4.10 and [27], Proposition 4.2.13. We also get that the Example 4.2.1 is completely disjoint from our present example and thus it is not cover by any other result of [27].

Based on 2.7 (b) and 2.6, we have the following equivalent form of 1.2. 
4.3. Basic Corollary. Suppose $A$ has compact factors. Let $E_{A}, g, \mathcal{A}_{g, 1, N}$, and $\mathcal{N}_{N}$ be as in 2.4. Then $\mathcal{N}_{N}$ is a projective $O_{E_{A}}\left[\frac{1}{N}\right]$-scheme which is a finite scheme over $\mathcal{A}_{g, 1, N}$.

4.4. Néron models. Let $\mathcal{K}$ be the field of fractions of an integral Dedekind ring $\mathcal{D}$. Let $Z_{\mathcal{K}}$ be a smooth, separated $\mathcal{K}$-scheme of finite type. We recall (cf. [3], page 12) that a Néron model of $Z_{\mathcal{K}}$ over $\mathcal{D}$ is a smooth, separated $\mathcal{D}$-scheme $Z$ of finite type that has $Z_{\mathcal{K}}$ as its generic fibre and that satisfies the following universal (Néron mapping) property:

for each smooth $\mathcal{D}$-scheme $Y$ and each $\mathcal{K}$-morphism $y_{\mathcal{K}}: Y_{\mathcal{K}} \rightarrow Z_{\mathcal{K}}$, there exists a unique morphism $y: Y \rightarrow Z$ of $\mathcal{D}$-schemes that extends $y_{\mathcal{K}}$.

A classical result of Néron says that any abelian variety over $\mathcal{K}$ has a Néron model over $\mathcal{D}$, cf. [24]. This result has an analogue for the case of torsors of smooth group schemes over $\mathcal{K}$ of finite type, cf. [3], Subsection 6.5, Corollary 4. On [3], page 15 it is stated that the importance of the notion of Néron models "seems to be restricted" to "torsors under group schemes". It was a deep insight of Milne which implicitly pointed out that Néron models are important in the study of Shimura varieties, cf. [18], Definitions 2.1, 2.2, 2.5, and 2.9. In this Subsection we bring to a concrete fruition Milne's insights: we will use 4.3 and [31] to provide large classes of projective varieties over certain $\mathcal{K}$ 's which have projective Néron models and which often do not admit finite maps into abelian varieties over $\mathcal{K}$. For the rest of the paper we will use the notations of $\S 1,2.4$, and 2.5.

4.4.1. Proposition. We assume that $A$ has compact factors and a principal polarization $\lambda_{A}$, that $O_{E_{A}}\left[\frac{1}{N}\right]$ is an étale $\mathbb{Z}\left[\frac{1}{N}\right]$-algebra, and that the $O_{E_{A}}\left[\frac{1}{N}\right]$-scheme $\mathcal{N}_{N}$ of 2.4 is smooth. Then $\mathcal{N}_{N}$ is the Néron model of $\mathcal{N}_{N E_{A}}=\operatorname{Sh}\left(H_{A}, X_{A}\right) / K_{A}(N)$ over $O_{E_{A}}\left[\frac{1}{N}\right]$.

Proof: Let $Y$ be a smooth $O_{E_{A}}\left[\frac{1}{N}\right]$-scheme. Let $y_{E_{A}}: Y_{E_{A}} \rightarrow \mathcal{N}_{N E_{A}}$ be a morphism of $E_{A}$-schemes. Let $U$ be an open subscheme of $Y$ such that it contains $Y_{E_{A}}$ and $y_{E_{A}}$ extends uniquely to a morphism $y_{U}: U \rightarrow \mathcal{N}_{N}$. As the $O_{E_{A}}\left[\frac{1}{N}\right]$-scheme $\mathcal{N}_{N}$ is projective (cf. 4.3), we can assume that the complement of $U$ in $Y$ has codimension at most 2 in $Y$.

Let $\left(B_{U}, \lambda_{B_{U}}\right):=y_{U}^{*}\left(\mathcal{B}, \lambda_{\mathcal{B}}\right)$. The abelian scheme $B_{U}$ extends to an abelian scheme $B_{Y}$ over $Y$ (cf. [31], Theorem 1.3) in an unique way (cf. [18], Corollary 2.12). Also $\lambda_{B_{U}}$ extends uniquely to a principal polarization $\lambda_{B_{Y}}$ of $B_{Y}$, cf. [18], Proposition 2.14. Obviously, the level- $N$ symplectic similitude structure of $\left(B_{U}, \lambda_{B_{U}}\right)$ extends uniquely to a level- $N$ symplectic similitude structure of $\left(B_{Y}, \lambda_{B_{Y}}\right)$. Thus the composite of $y_{U}$ with the finite morphism $\mathcal{N}_{N} \rightarrow \mathcal{A}_{g, 1, N}$ extends uniquely to a morphism $z: Y \rightarrow \mathcal{A}_{g, 1, N}$. As $Y$ is normal and as the morphism $\mathcal{N}_{N} \rightarrow \mathcal{A}_{g, 1, N}$ is finite, $z$ factors uniquely through a morphism $y: Y \rightarrow \mathcal{N}_{N}$. Obviously $y$ extends $y_{U}$ and thus also $y_{E_{A}}$. From this and the uniqueness of $y$ and $y_{U}$, we get that $\mathcal{N}_{N}$ satisfies the Néron mapping property. Thus $\mathcal{N}_{N}$ is the Néron model of $\mathcal{N}_{N E_{A}}=\operatorname{Sh}\left(H_{A}, X_{A}\right) / K_{A}(N)$ over $O_{E_{A}}\left[\frac{1}{N}\right]$.

4.4.2. Remark. If $N$ has many prime divisors, then $K_{A}(N)$ is a small enough compact, open subgroup of $H_{A}\left(\mathbb{A}_{f}\right)$ and thus $\operatorname{Sh}\left(H_{A}, X_{A}\right)_{\mathbb{C}} / K_{A}(N)$ is a projective, smooth $\mathbb{C}$-scheme of general type (see [17], $§ 2$, Subsection 1.2). Thus $\mathcal{N}_{N}$ is not among the Néron models studied in [3]. If the albanese variety of each connected component $\mathcal{C}$ of $\operatorname{Sh}\left(H_{A}, X_{A}\right)_{\mathbb{C}} / K_{A}(N)$ is trivial, then $\operatorname{Sh}\left(H_{A}, X_{A}\right) / K_{A}(N)$ is not a finite scheme over an abelian variety over

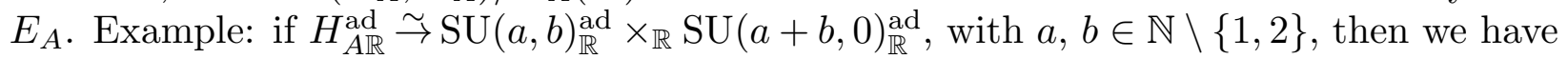


$H^{1,0}(\mathcal{C}(\mathbb{C}), \mathbb{C})=0$ (cf. [26], Theorem 2, $\left.2.8(\mathrm{i})\right)$ and thus the albanese variety of $\mathcal{C}$ is trivial; thus the connected components of the projective $E_{A}$-scheme $\operatorname{Sh}\left(H_{A}, X_{A}\right) / K_{A}(N)$ are not finite schemes over torsors of smooth groups over $E_{A}$. This remark was hinted at in [31].

4.5. Example. We assume that $A$ has compact factors, that $N \in 6 \mathbb{N}$, and that the Zariski closure $H_{A, N}$ of $H_{A}$ in $\mathrm{GL}_{L_{A}\left[\frac{1}{N}\right]}$ is a reductive group scheme over $\mathbb{Z}\left[\frac{1}{N}\right]$. We also assume that if $\left(H_{A}^{\mathrm{ad}}, X_{A}^{\mathrm{ad}}\right)$ has a simple factor of $A_{n}$ type, then either all prime factors of $n+1$ divide $N$ or the degree of the isogeny $H_{A}^{\mathrm{sc}} \rightarrow H_{A}^{\text {der }}$ divides $N .^{1}$ Let $p \in \mathbb{N}$ be an arbitrary prime that does not divide $N$; thus $p \geq 5$. Let $\mathbb{Z}_{(p)}$ be the localization of $\mathbb{Z}$ with respect to $p$. As $H_{A, N_{\mathbb{Z}_{(p)}}}$ is a reductive group scheme over $\mathbb{Z}_{(p)}$, the field $E_{A}$ is unramified over $p$ (cf. [19], Corollary 4.7 (a)). Thus the normalization $E_{A(p)}$ of $\mathbb{Z}_{(p)}$ in $E_{A}$ is a finite, étale $\mathbb{Z}_{(p)}$-algebra. This implies that $O_{E_{A}}\left[\frac{1}{N}\right]$ is a finite, étale $\mathbb{Z}\left[\frac{1}{N}\right]$-algebra. In the next two paragraphs we check that $\mathcal{N}_{N}$ is a smooth $O_{E_{A}}\left[\frac{1}{N}\right]$-scheme.

As $N \geq 3$, the intersection $\operatorname{GSp}\left(W_{A}, \psi_{A}\right)(\mathbb{Q}) \cap K(N)$ is a torsion free group. If $h \in \operatorname{GSp}\left(W_{A}, \psi_{A}\right)\left(\mathbb{A}_{f}\right)$, then the group $\operatorname{GSp}\left(W_{A}, \psi_{A}\right)(\mathbb{Q}) \cap h K(N) h^{-1}$ is isomorphic to $\operatorname{GSp}\left(W_{A}, \psi_{A}\right)(\mathbb{Q}) \cap K(N)$ and thus is a torsion free group. Thus $H_{A}(\mathbb{Q}) \cap h K_{A}(N) h^{-1}$ is also a torsion free group. But $\operatorname{Sh}\left(H_{A}, X_{A}\right)_{\mathbb{C}} / K_{A}(N)$ is a disjoint union of quotients of connected hermitian symmetric domains by arithmetic subgroups of the form $H_{A}(\mathbb{Q}) \cap$ $h K_{A}(N) h^{-1}$ with $h \in H_{A}\left(\mathbb{A}_{f}\right)$ and thus by torsion free arithmetic subgroups. This implies that the natural morphism $\operatorname{Sh}\left(H_{A}, X_{A}\right)_{\mathbb{C}} \rightarrow \operatorname{Sh}\left(H_{A}, X_{A}\right)_{\mathbb{C}} / K_{A}(N)$ is a pro-étale cover. Thus $\operatorname{Sh}\left(H_{A}, X_{A}\right) / K_{A}(N)$ is a smooth $E_{A}$-scheme. We consider the projective

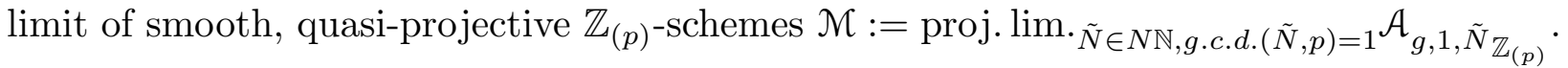

We can identify $\mathcal{M}_{\mathbb{Q}}=\operatorname{Sh}\left(\operatorname{GSp}\left(W_{A}, \psi_{A}\right), S_{A}\right) / \operatorname{GSp}\left(L_{A}\right)\left(\mathbb{Z}_{p}\right)$. Let $\mathcal{N}^{(p)}:=\mathcal{N}_{A}^{(p)}$ be the normalization of $\mathcal{M}$ in (the ring of fractions of) $\operatorname{Sh}\left(H_{A}, X_{A}\right) / H_{A, N}\left(\mathbb{Z}_{p}\right)$; the $E_{A}$-scheme $\mathcal{N}_{E_{A}}^{(p)}=\operatorname{Sh}\left(H_{A}, X_{A}\right) / H_{A, N}\left(\mathbb{Z}_{p}\right)$ is a pro-étale cover of $\mathcal{N}_{N E_{A}}=\operatorname{Sh}\left(H_{A}, X_{A}\right) / K_{A}(N)$.

From proof of [30], Proposition 3.4.1 we get that $\mathcal{N}^{(p)}$ is a pro-étale cover of $\mathcal{N}_{N E_{A(p)}}$ (the previous paragraph implies that conditions (i) and (ii) of loc. cit. hold in the context of $\mathcal{M}$ and $\left.\mathcal{N}^{(p)}\right)$. As $p \geq 5$, from [30], Subsubsections 3.4.1, 3.2.12, and 6.4.1 we get that $\mathcal{N}^{(p)}$ is the integral canonical model of the Shimura triple $\left(H_{A}, X_{A}, H_{A, N}\left(\mathbb{Z}_{p}\right)\right)$ as defined in [30], Subsubsections 3.2.6 and 3.2.3 6). Thus $\mathcal{N}^{(p)}$ is a regular, formally smooth $E_{A(p)^{-}}$

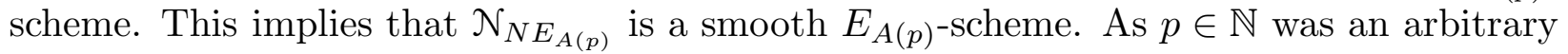
prime that does not divide $N$, we conclude that $\mathcal{N}_{N}$ is a smooth $O_{E_{A}}\left[\frac{1}{N}\right]$-scheme.

Thus $\mathcal{N}_{N}$ is a Néron model of $\mathcal{N}_{N E_{A}}$ over $O_{E_{A}}\left[\frac{1}{N}\right]$, cf. 4.4.1.

4.6. Remarks. (a) Either [15], $\S 5$ or [33] can be used to provide many examples similar to the one of 4.5 but with $N$ relatively prime to either 2 or 3 .

(b) We refer to 4.5; thus the prime $p$ is at least 5. As $\mathcal{N}_{N}$ is a projective, smooth $O_{E_{A}}\left[\frac{1}{N}\right]$-scheme, $\mathcal{N}^{(p)}$ is a pro-étale cover of a projective, smooth $E_{A(p)}$-scheme. This validates the erroneous [30], Remark 6.4.1.12) for the case of Shimura pairs $(G, X)$ of abelian type that have compact factors. In other words, if the group $G_{\mathbb{Q}_{p}}$ is unramified,

1 This condition is not truly needed. It is inserted only to avoid the error which was made in the b) part of [30], Theorem 6.2.2 and which is eliminated in [33]. 
then the scheme $\operatorname{Sh}_{p}(G, X)$ proved to exist in [30], Theorem 6.4.1 is a pro-étale cover of a smooth, projective scheme over the normalization of $\mathbb{Z}_{(p)}$ in $E(G, X)$. Based on [30], Subsection 6.8 and Subsubsections 6.8.1 and 6.8.2 a), in the last sentence one can replace "abelian type" by "preabelian type". Implicitly, this validates [30], Subsubsection 6.4.11 for all Shimura pairs $(G, X)$ of preabelian type that have compact factors.

\section{References}

[1] W. Baily and A. Borel, Compactification of arithmetic quotients of bounded symmetric domains, Ann. of Math. (2) 84 (1966), no. 3, pp. 442-528.

[2] A. Borel and Harish-Chandra, Arithmetic subgroups of algebraic groups, Ann. of Math. (2) 75 (1962), no. 3, pp. 485-535.

[3] S. Bosch, W. Lütkebohmert, and M. Raynaud, Néron models, Ergebnisse der Mathematik und ihrer Grenzgebiete (3), Vol. 21, Springer-Verlag, 1990.

[4] N. Bourbaki, Lie groups and Lie algebras, Chapters 4-6, Springer-Verlag, 2002.

[5] P. Berthelot and A. Ogus, F-crystals and de Rham cohomology.I, Invent. Math. 72 (1983), no. 2, pp. 159-199.

[6] P. Deligne, Travaux de Shimura, Séminaire Bourbaki, 23ème année (1970/71), Exp. No. 389, pp. 123-165, Lecture Notes in Math., Vol. 244, Springer-Verlag, 1971.

[7] P. Deligne, Variétés de Shimura: interprétation modulaire, et techniques de construction de modèles canoniques, Automorphic forms, representations and $L$ functions (Oregon State Univ., Corvallis, OR, 1977), Part 2, pp. 247-289, Proc. Sympos. Pure Math., Vol. 33, Amer. Math. Soc., Providence, RI, 1979.

[8] P. Deligne, Hodge cycles on abelian varieties, Hodge cycles, motives, and Shimura varieties, Lecture Notes in Math., Vol. 900, pp. 9-100, Springer-Verlag, 1982.

[9] M. Demazure, A. Grothendieck, et al., Schémas en groupes, Vol. II, Lecture Notes in Math., Vol. 152, Springer-Verlag, 1970.

[10] G. Faltings and C.-L. Chai, Degeneration of abelian varieties, Ergebnisse der Mathematik und ihrer Grenzgebiete (3), Vol. 22, Springer-Verlag, 1990.

[11] J.-M. Fontaine, Le corps des périodes p-adiques, Périodes p-adiques (Bures-surYvette, 1988), pp. 103-112, J. Astérisque 223, Soc. Math. de France, Paris, 1994.

[12] J.-M. Fontaine, Représentations p-adiques semi-stables, Périodes $p$-adiques (Buressur-Yvette, 1988), J. Astérisque 223, pp. 113-184, Soc. Math. de France, Paris, 1994.

[13] A. Grothendieck, Éléments de géométrie algébrique. IV. Étude locale des schémas et des morphismes de schéma (Quatrième Partie), Inst. Hautes Études Sci. Publ. Math., Vol. 32, 1967.

[14] R. Hartshorne, Algebraic geometry, Grad. Texts in Math. 52, Springer-Verlag, 1977.

[15] R. E. Kottwitz, Points on some Shimura Varieties over finite fields, J. of Amer. Math. Soc. 5 (1992), no. 2, pp. 373-444.

[16] H. Matsumura, Commutative algebra. Second edition, The Benjamin/Cummings Publishing Co., Inc., 1980. 
[17] J. S. Milne, Canonical models of (mixed) Shimura varieties and automorphic vector bundles, Automorphic Forms, Shimura varieties and L-functions, Vol. I (Ann Arbor, MI, 1988), pp. 283-414, Perspectives in Math., Vol. 10, Acad. Press, 1990.

[18] J. S. Milne, The points on a Shimura variety modulo a prime of good reduction, The Zeta function of Picard modular surfaces, pp. 153-255, Univ. Montréal, Montreal, Quebec, 1992.

[19] J. S. Milne, Shimura varieties and motives, Motives (Seattle, WA, 1991), Part 2, pp. 447-523, Proc. Symp. Pure Math., Vol. 55, Amer. Math. Soc., Providence, RI, 1994.

[20] J. S. Milne, Descent for Shimura varieties, Mich. Math. J. 46 (1999), no. 1, pp. 203-208.

[21] Y. Morita, On potential good reduction of abelian varieties, J. Fac. Sci. Univ. Tokyo Sect. I A Math. 22 (1975), no. 3, pp. 437-447.

[22] D. Mumford, J. Fogarty, and F. Kirwan, Geometric invariant theory. Third enlarged edition, Ergebnisse der Mathematik und ihrer Grenzgebiete (2), Vol. 34, Springer-Verlag, 1994.

[23] D. Mumford, Abelian varieties, Tata Inst. of Fund. Research Studies in Math., No. 5, Published for the Tata Institute of Fundamental Research, Bombay; Oxford Univ. Press, London, 1970 (reprinted 1988).

[24] A. Néron, Modèles minimaux des variétés abéliennes, Inst. Hautes Études Sci. Publ. Math., Vol. 21, 1964.

[25] R. Noot, Abelian varieties with l-adic Galois representations of Mumford's type, J. reine angew. Math. 519 (2000), pp. 155-169.

[26] R. Parthasarathy, Holomorphic forms in $\Gamma \backslash G / K$ and Chern classes, Topology 21 (1982), no. 2, pp. 157-178.

[27] F. Paugam, Galois representations, Mumford-Tate groups and good reduction of abelian varieties, Math. Ann. 329 (2004), no. 1, pp. 119-160. Erratum: Math. Ann. 332 (2004), no. 4, p. 937.

[28] T. A. Springer, Reductive groups, Automorphic forms, representations and $L$ functions (Oregon State Univ., Corvallis, Ore., 1977), Part 1, pp. 3-27, Proc. Sympos. Pure Math., Vol. 33, Amer. Math. Soc., Providence, RI, 1979.

[29] J. Tits, Classification of algebraic semisimple groups, Algebraic Groups and Discontinuous Subgroups (Boulder, CO, 1965), pp. 33-62, Proc. Sympos. Pure Math., Vol. 9, Amer. Math. Soc., Providence, RI, 1966.

[30] A. Vasiu, Integral canonical models of Shimura varieties of preabelian type, Asian J. Math. 3 (1999), no. 2, pp. 401-518.

[31] A. Vasiu, A purity theorem for abelian schemes, Mich. Math. J. 54 (2004), no. 1, pp. $71-81$.

[32] A. Vasiu, The Mumford-Tate Conjecture and Shimura Varieties, Part I, math.NT/0212066.

[33] A. Vasiu, Integral canonical models of unitary Shimura varieties, math.NT/0505507.

Adrian Vasiu, Email: adrian@math.arizona.edu

Address: University of Arizona, Department of Mathematics, 617 North Santa Rita, P.O. Box 210089, Tucson, AZ-85721, U.S.A. 